\title{
Axial tubule junctions control rapid calcium signaling in atria
}

\author{
Sören Brandenburg, ${ }^{1,2}$ Tobias Kohl, ${ }^{1,2}$ George S.B. Williams, ${ }^{3}$ Konstantin Gusev, ${ }^{1,2}$ Eva Wagner, ${ }^{1,2,4}$ Eva A. Rog-Zielinska, ${ }^{5}$ \\ Elke Hebisch, ${ }^{6}$ Miroslav Dura, ${ }^{1,2}$ Michael Didié, ${ }^{1,2}$ Michael Gotthardt, ${ }^{4,7}$ Viacheslav O. Nikolaev, ${ }^{1,2,4}$ Gerd Hasenfuss, ${ }^{1,2,4}$ \\ Peter Kohl, ${ }^{5,8}$ Christopher W. Ward, ${ }^{3}$ W. Jonathan Lederer, ${ }^{3}$ and Stephan E. Lehnart ${ }^{1,2,4}$ \\ ${ }^{1}$ Heart Research Center Cöttingen and ${ }^{2}$ Clinic of Cardiology and Pneumology, University Medical Center Cöttingen, Cöttingen, Cermany. ${ }^{3}$ BioMET, Center for Biomedical Engineering and Technology, \\ University of Maryland School of Medicine, Baltimore, Maryland, USA. ${ }^{4}$ Cerman Centre for Cardiovascular Research (DZHK), Göttingen, Germany. ${ }^{5}$ mmperial College London, National Heart and Lung Institute, \\ London, United Kingdom. ${ }^{6}$ Department of NanoBiophotonics, Max-Planck Institute for Biophysical Chemistry, Göttingen, Germany. ${ }^{7}$ Max Delbrück Center for Molecular Medicine, Berlin, Germany. \\ ${ }^{8}$ University Heart Centre, Faculty of Medicine, University of Freiburg, Freiburg im Breisgau, Cermany.
}

\begin{abstract}
The canonical atrial myocyte (AM) is characterized by sparse transverse tubule (TT) invaginations and slow intracellular $\mathrm{Ca}^{2+}$ propagation but exhibits rapid contractile activation that is susceptible to loss of function during hypertrophic remodeling. Here, we have identified a membrane structure and $\mathrm{Ca}^{2+}$-signaling complex that may enhance the speed of atrial contraction independently of phospholamban regulation. This axial couplon was observed in human and mouse atria and is composed of voluminous axial tubules (ATs) with extensive junctions to the sarcoplasmic reticulum (SR) that include ryanodine receptor $\mathbf{2}$ (RyR2) clusters. In mouse AM, AT structures triggered Ca ${ }^{2+}$ release from the SR approximately 2 times faster at the AM center than at the surface. Rapid $\mathrm{Ca}^{2+}$ release correlated with colocalization of highly phosphorylated RyR2 clusters at AT-SR junctions and earlier, more rapid shortening of central sarcomeres. In contrast, mice expressing phosphorylation-incompetent RyR2 displayed depressed AM sarcomere shortening and reduced in vivo atrial contractile function. Moreover, left atrial hypertrophy led to AT proliferation, with a marked increase in the highly phosphorylated RyR2-pS2808 cluster fraction, thereby maintaining cytosolic $\mathrm{Ca}^{2+}$ signaling despite decreases in RyR2 cluster density and RyR2 protein expression. AT couplon "super-hubs" thus underlie faster excitation-contraction coupling in health as well as hypertrophic compensatory adaptation and represent a structural and metabolic mechanism that may contribute to contractile dysfunction and arrhythmias.
\end{abstract}

\section{Introduction}

Electrical and contractile dysfunction of the atria are frequent components of cardiac disease development, often culminating in atrial fibrillation (AF), a leading cause of ischemic stroke, which is predicted to triple in prevalence by 2050 (1). Among the risk factors associated with atrial dysfunction, hypertension is present in $60 \%$ to $80 \%$ of European patients (2). Using rapid pacing, key aspects of atrial remodeling and AF were reproduced in large animal models: whereas electrical and ionic changes occurred within minutes, intracellular $\mathrm{Ca}^{2+}$ overload was normal by 48 hours (3). Recently, high atrial pacing rates were shown to induce $\mathrm{Ca}^{2+}$-signaling silencing, preventing intracellular $\mathrm{Ca}^{2+}$ overload, albeit at the cost of depressed contractile function (4). Hypertrophy with depressed contractile function occurred as early as 2 days after pacing, in the absence of fibrosis or dilation in canine atria (5). These studies support the therapeutically relevant notion that electrical and metabolic disease mechanisms may cause contractile atrial dysfunction relatively early, preceding AF.

\section{Related Commentary: p. 3731}

Conflict of interest: The authors have declared that no conflict of interest exists. Submitted: April 27, 2016; Accepted: August 9, 2016.

Reference information: J Clin Invest. 2016;126(10):3999-4015. doi:10.1172/JCI88241.
Atrial myocytes (AM) are distinguished from ventricular myocytes (VM) by their smaller diameter, which may explain why no or few transverse tubules (TT) could support adequate AM function (6-8). In contrast, VMs are characterized by high TT density and regularity in normal hearts, a feature lost in heart failure (HF) (9, 10). Given the regular presence of TTs at sarcomeric Z-lines, TT density is thought to determine uniform $\mathrm{Ca}^{2+}$ release in VMs (11). Paradoxically, despite sparse irregular TT components, contractile activation of atrial muscle from small and large animal species occurs faster than in ventricular tissue (12). Hence, there is a gap in understanding the mechanisms that underlie rapid activation of atrial contraction. In addition, even though atrial dysfunction and arrhythmias are recognized as a leading cause of cardiac disease burden, fundamental details about the subcellular mechanisms involved remain unclear (13).

In the absence of abundant $\mathrm{TT}$ membrane invaginations, AMs are thought to initiate excitation-contraction coupling (ECC) through $\mathrm{Ca}^{2+}$ release units (CRUs) at the surface sarcolemma, each including approximately 6 L-type $\mathrm{Ca}^{2+}$ channels (LCCs/Cav1.2) opposite a cluster of approximately $50 \mathrm{RyR} 2 \mathrm{Ca}^{2+}$ release channels, separated by a subspace of approximately $15 \mathrm{~nm}$ width $(14,15)$. In this model, $\mathrm{Ca}^{2+}$ transients are activated through subsarcolemmal CRUs, leading initially to peripheral elevation of $\mathrm{Ca}^{2+}$, which travels toward the AM center through propagated $\mathrm{Ca}^{2+}$-induced $\mathrm{Ca}^{2+}$ 
A

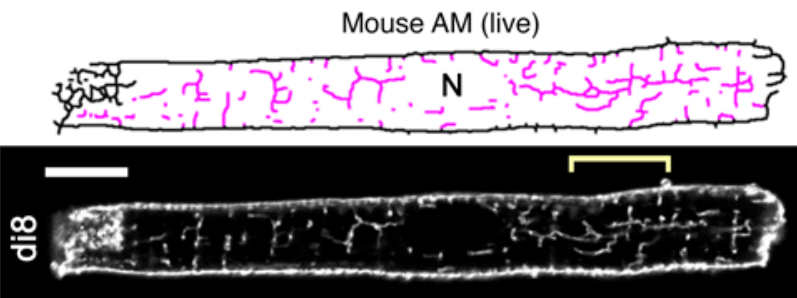

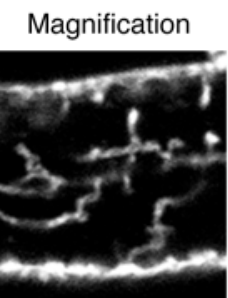

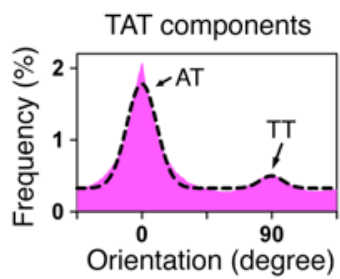

B

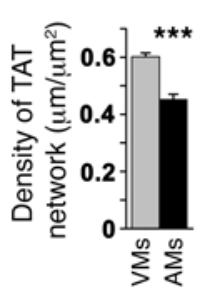

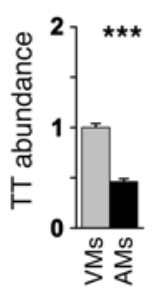

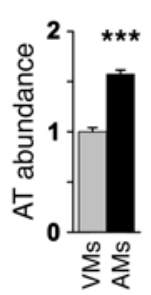

C
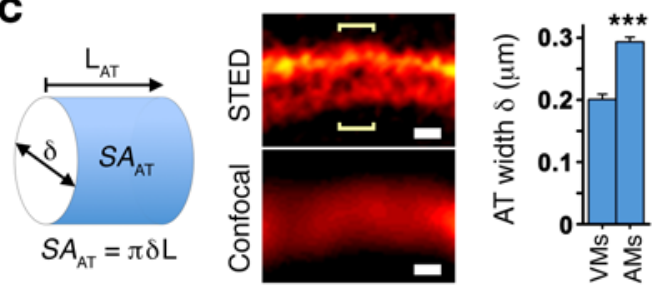

D

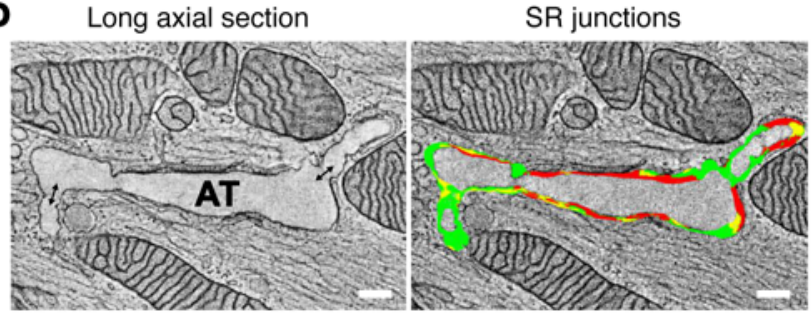

E Cross section

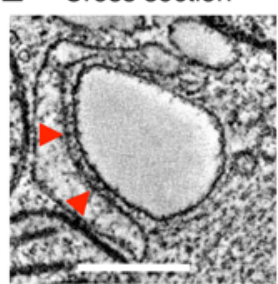

$\mathbf{F}$
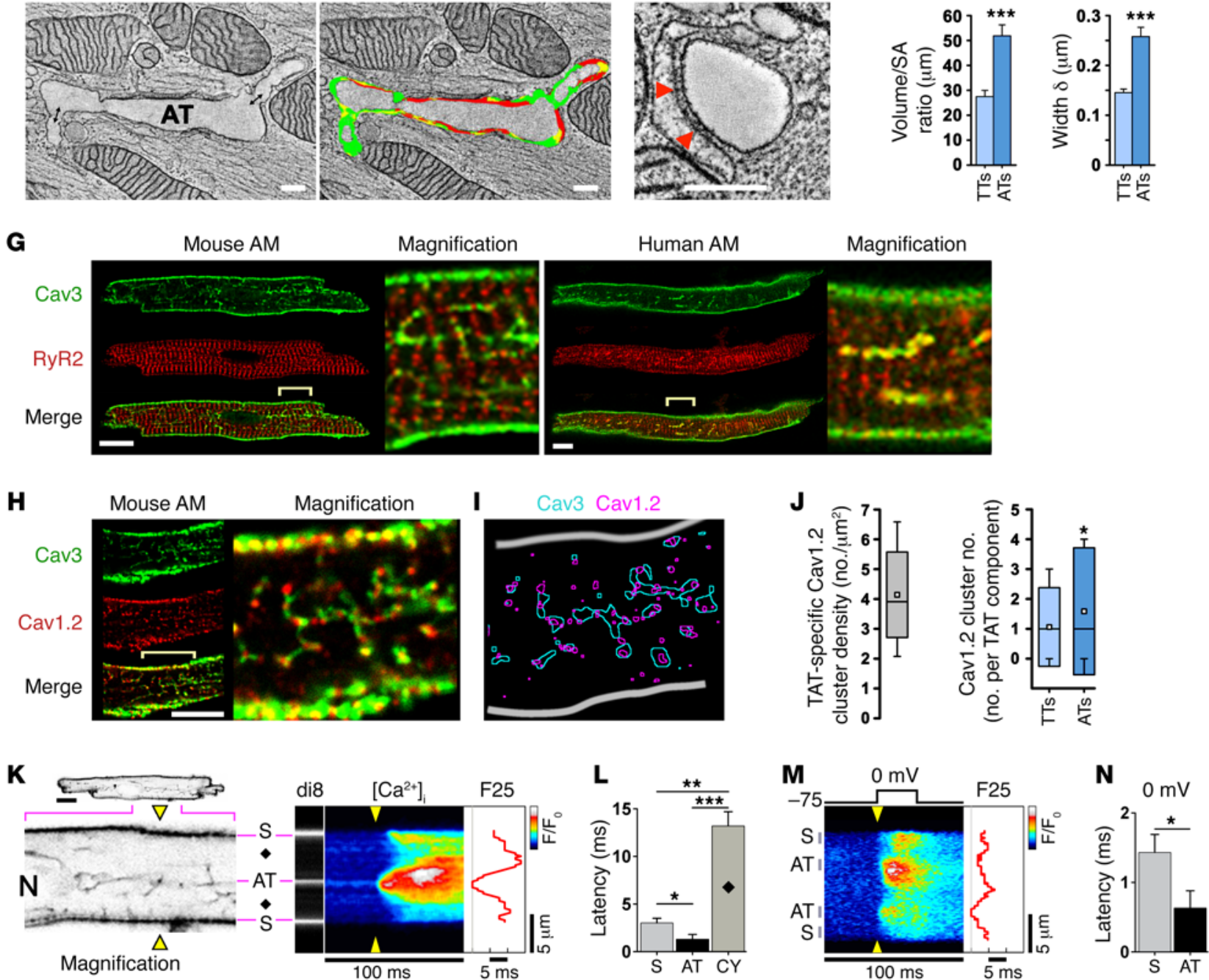
Figure 1. Abundant AT structures rapidly activate $\mathrm{Ca}^{2+}$ release. (A) Confocal live imaging of di-8-ANEPPS-stained (di8) TAT structures visualized as skeletons (pink). N, nucleus. Scale bar: $10 \mu \mathrm{m}$. TAT component orientations (histogram) and Gaussian fitting show abundant AT $\left(0^{\circ}\right)$ versus sparse TT $\left(90^{\circ}\right)$ components (binning $\left.\pm 20^{\circ}\right) . n=36$ AMs. (B) Comparison of ventricular versus atrial TAT network length normalized to cell area; TT and AT component abundance. $n=36 \mathrm{AMs}$ and $25 \mathrm{VMs}$. (C) Illustration conceptualizing AT width $(\delta)$ measurements and calculated surface area (SA). L, length. AT width was determined from local STED signal distributions of optical cross sections (brackets) and is summarized in the bar graph. Scale bars: $200 \mathrm{~nm} . n=27$ VMs, 30 AMs. (D) Double-headed arrows indicate potential AT-TT connections; ET images and segmentation of longitudinally sectioned and (E) cross-sectioned AT structures. Scale bars: $200 \mathrm{~nm}$. Color legend - red, AT-SR junctions $\leq 15 \mathrm{~nm}$ in gap width and containing RyR2 densities; yellow, AT-SR junctions $\leq 20 \mathrm{~nm}$ in gap width but lacking RyR2 densities; green, membrane area with no apparent junctions. AT-TT junction; for color rendering, see Supplemental Figure 4. Arrows indicate exemplary electron densities compatible with RyR2 channels. (F) Bar graphs comparing TT versus AT volume/surface area ratio and width. $n=13$ TTs, 23 ATs. Data are representative of 3 hearts. (G and $\mathbf{H}$ ) Confocal images of Cav3-, RyR2-, and Cav1.2-coimmunostained mouse and human AMs. Robust Cav3-labeled AT structures in human and mouse AMs. Scale bars: $10 \mu \mathrm{m}$, magnification $\times 4$. Yellow brackets indicate regions magnified. (I) Image segmentation. (J) Box plots summarizing TAT-specific Cav1.2 cluster density; component-specific Cav1.2 cluster numbers. White boxes indicate the mean; boxes represent the $50^{\text {th }}$ percentile and lower and upper SD, and whiskers represent the $10^{\text {th }}$ and $90^{\text {th }}$ percentiles. $n=17 \mathrm{AMs}$. (K) Confocal visualization (negative contrast) of AT structures for transversal line scanning (yellow triangles) of intracellular $\mathrm{Ca}^{2+}$ (fluo-4) magnification $\times 4$ : field potential-evoked $\mathrm{Ca}^{2+}$ transient activated via AT and subsurface (S) structures; black diamonds, off-membrane $\mathrm{CY} ; \mathrm{F} 25, \mathrm{Ca}^{2+}$ signal onset at $25 \%$ signal amplitude; F/ $F_{0}$, Normalized fluorescence intensity ratio indicated by look-up-table; N, nucleus. (L) Relative latency of early $\mathrm{Ca}^{2+}$ signal upstroke $(\mathrm{dF} / \mathrm{dt})$ for the indicated locations. Data are representative of 19 AMs. (M) Voltage-clamped AMs (1 $\mathrm{mM}$ EGTA) during $\mathrm{Ca}^{2+}$ transient activation. $\mathrm{F} 25, \mathrm{Ca}^{2+}$ signal onset during -75 to $0 \mathrm{mV}$ depolarization. (N) Latency difference of $\mathrm{Ca}^{2+}$ signal upstroke (dF/dt). $n=15$ AMs. ${ }^{*} P<0.05,{ }^{* *} P<0.01$, and ${ }^{* *} P<0.001$, by Student's $t$ test $(\mathbf{A}-\mathbf{F}, \mathbf{K}-\mathbf{N})$ and Mann-Whitney $U$ test (J).

release (CICR) within approximately $100 \mathrm{~ms}(4,7)$. Hence, central $\mathrm{Ca}^{2+}$ release should be significantly delayed, consistent with observations of $\mathrm{U}$-shaped atrial $\mathrm{Ca}^{2+}$ release activation in transversal line scans of AMs $(4,16,17)$. Such slow atrial ECC is at odds, though, with the rapid mechanical activation dynamics of atrial muscle (12). We hypothesize that AM-specific components of the transverse axial tubule (TAT) system combine molecular functions with rapid $\mathrm{Ca}^{2+}$ signal activation in a highly localized manner in AMs.

Our investigations were made possible by recent methodological advances, which provide high-quality samples for imaging of intact AM membrane structures $(9,18)$. While intracellular TAT structures are generally robust and functional throughout AMs, as described below, it was remarkable to observe large axial tubule (AT) structures connected through a sparse pattern of TTs. The unusually voluminous ATs are functionally coupled to RyR2 clusters in axial couplons, which form extensive junctional complexes that allow the rapid central $\mathrm{Ca}^{2+}$ signaling that we investigated by high-resolution live-cell imaging, electron tomography (ET), patch-clamp recordings, and mathematical modeling. Unexpectedly, RyR2 clusters colocalized with AT membranes were highly phosphorylated in situ by protein kinase A (PKA) and $\mathrm{Ca}^{2+}$-calmodulin-dependent protein kinase (CaMK) and hence clearly dis- tinguishable from minimally phosphorylated nonjunctional RyR2 clusters. This correlated with substantially earlier $\mathrm{Ca}^{2+}$ release from AT-associated RyR2 clusters and faster central sarcomere shortening, leading to a new model of atrial contractile regulation.

\section{Results}

AMs contain cell-wide TAT membrane networks. Although earlier electron microscopy-based (EM-based) representations of AMs (6), recent confocal data, and computational models (19) suggest that there are few, if any, TT invaginations, experimental studies have increasingly highlighted the presence of atrial TAT networks in several mammalian species, including humans (20-22). To clarify this apparent contradiction, we used membrane-preserving workflows developed for high-resolution live-cell imaging $(9$, 18) that revealed abundant TAT membrane structures in murine AMs (Figure 1A and Supplemental Figure 1A; supplemental material available online with this article; doi:10.1172/JCI88241DS1). We identified intact TAT networks in 100\% of the AMs examined $(n=29)$, thus confirming the presence of preserved membrane structures. Using skeletonized images, we visualized individual TAT components and analyzed their orientations (Figure 1A, magenta). In confocal sections, the cell-wide TAT network density in AMs was lower (by $24 \%$ ) than in VMs (isolated from the same hearts), attributable chiefly to a significantly lower presence of TT components (Figure 1B), which dominate TAT networks in VMs (Supplemental Figure 1B). Despite smaller dimensions (Supplemental Figure 2) and a low TT abundance, mouse AMs contained significantly more ATs than VMs (Figure 1B). Thus, while cell type-specific differences in the abundance of TTs explain a moderately lower TAT network density, AMs showed a significantly higher abundance of AT components than did VMs.

Since confocal imaging suggested unusually large AT dimensions (Figure 1C, illustration), we used live-cell stimulated emission depletion (STED) super-resolution microscopy (nanoscopy) to assess the width of intact ATs and TTs (Supplemental Figure 3). AT width $(293.3 \pm 7.7 \mathrm{~nm})$ was significantly greater than TT width in AMs (Supplemental Table 1) and approximately 50\% larger in AMs than in VMs (Figure 1C and Supplemental Table 1). This was further investigated using ET and 3D segmentation (23) of semithick sections $(280 \mathrm{~nm})$ of high-pressure-frozen AMs (Supplemental Figure 4). ET confirmed the presence of voluminous ATs containing multiple junctions with the sarcoplasmic reticulum (SR) (Figure 1D). The color rendering in Supplemental Figure 4 highlights extensive AT-SR junctions with a gap width of $15 \mathrm{~nm}$ or less that contained RyR2 densities (red) covering large regions of the AT surface area (Figure 1, D and E). While not perfectly circular in the cross section, we observed no difference in the mean eccentricity of ATs and TTs $(0.62 \pm 0.05, n=11$ and $0.64 \pm 0.04$, $n=9$, respectively; $P=0.7)$. The measured volume/surface area ratio and width were significantly higher for ATs than for TTs in AMs (Figure 1F), consistent with the STED measurements. These independent techniques confirmed the presence of central-axial membrane structures of unusually large size and surface area that were distinct from those of VMs, with extensive SR junctions.

AT structures couple excitation to rapid intracellular $\mathrm{Ca}^{2+}$ release. TT invaginations, continuous with the surface membrane, contain LCCs juxtapositioned with RyR2 clusters in the SR, and similar 
A
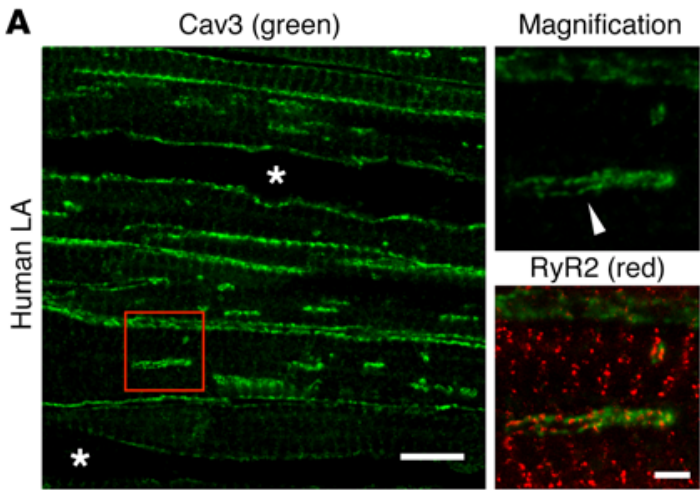

B

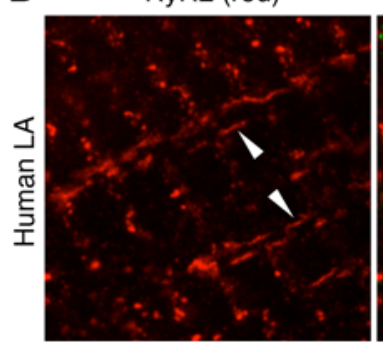

Cav3 (green)

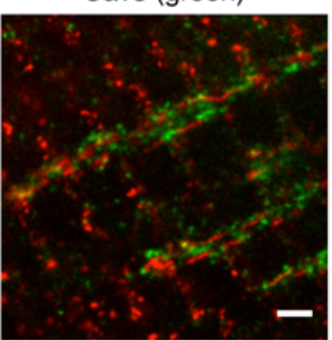

structural features were evident for ATs in our EM AM data (Figure 1, D and E). To investigate the subcellular relations between RyR2 channels and AT structures, we immunolocalized RyR2 and caveolin 3 (Cav3). While colocalization of Cav3 and RyR2 is generally considered to indicate the presence of CRUs at SR contact sites (24), we observed no regular striated distribution due to sparse TT elements in AMs (Figure 1G). Instead, robust Cav3-positive AT structures were abundant in AMs from both mouse and human hearts (Figure 1G). Furthermore, Cav1.2 channel clusters were frequent and regularly associated with AT structures (Figures 1, H and I). Analysis of Cav1.2 clusters confirmed a substantial TAT-specific density, and the number of component-specific Cav1.2 clusters was significantly higher in AT components than in TT components (Figure 1J). Thus, central RyR2 clusters, separated by a subsurface gap from the peripheral RyR2 clusters described previously (19), colocalize in AMs with abundant AT structures that contain a high number of Cav1.2 clusters.

For AT structure-function analysis, we measured $\mathrm{Ca}^{2+}$ transients in AM using fluo-4 and the membrane-intercalculating dye di-8-ANEPPS using protocols established previously $(9,18)$. Transversal line scanning during electrical stimulation revealed robust $\mathrm{Ca}^{2+}$ rises, originating from AT sites (Figure 1K). Surprisingly, the spatiotemporal F25 profile (time point at which $25 \%$ of the maximal fluorescence is reached) indicated earlier $\mathrm{Ca}^{2+}$ transient onset at ATs than at surface (S) sarcolemmal sites (Figure $1 \mathrm{~K})$. To confirm this, we analyzed $\mathrm{Ca}^{2+}$ signal onset at $\mathrm{AT}, \mathrm{S}$, and off-membrane cytosolic (CY) locations by assessing the first derivative of fluorescence intensity $(\mathrm{dF} / \mathrm{dt})$ and determined the following latency profile: $\mathrm{AT}<\mathrm{S}<<\mathrm{CY}$ (Figure 1L). In contrast, imaging planes that excluded visible AT structures showed principally different, U-shaped $\mathrm{Ca}^{2+}$ transient onset patterns (data not shown). This result is consistent with the steep $\mathrm{S}<<\mathrm{CY}$ latency gradients we observed (Figure 1L) and agrees with previous observations of $\mathrm{U}$-shaped $\mathrm{Ca}^{2+}$ transients $(4,17,25)$. To reproduce early $\mathrm{Ca}^{2+}$ sig-

Figure 2. ATs and junctions with RyR2 clusters are common in human tissue. Dual-color STED nanoscopy of 2 human LA tissue sections coimmunostained for Cav3 and RyR2. (A) Tissue section shows AMs aligned with capillaries (asterisks indicate the lumens). Magnified images (of red boxed area) show 1 Cav3-labeled AT membrane structure (arrowhead), which intersects with 6 transversal rows of RyR2 clusters delimited by the surface membrane on top. Scale bars: overview $10 \mu \mathrm{m}$; magnifications, $2 \mu \mathrm{m}$. (B) Intracellular high-power magnifications showing 2 parallel, axially aligned RyR2 cluster tracks, perpendicularly intersecting 6 transversal RyR2 rows. Arrowheads identify exemplary RyR2 clusters with typical axial couplon architectures. RyR2 clusters with elongated axial morphologies and Cav3-labeled structures are tightly spaced next to each other, indicating a functional role of AT-associated SR junctions. STED images are representative of 5 human LA samples. Scale bar: $2 \mu \mathrm{m}$.

nal onset at AT structures under direct voltage control, we patch clamped AMs (1 mM EGTA in the patch; Figure 1M). Analysis of $\mathrm{Ca}^{2+}$ signal onset by $\mathrm{dF} / \mathrm{dt}$ during depolarization to $\mathrm{O} \mathrm{mV}$ confirmed the significant $\mathrm{AT}<\mathrm{S}$ latency (Figure $1 \mathrm{~N}$ ). In summary, electrically evoked $\mathrm{Ca}^{2+}$ transients originate deep inside AMs, from AT structures, and do so approximately 2 times earlier than $\mathrm{Ca}^{2+}$ transients on the surface membrane. The discovery of more rapid central $\mathrm{Ca}^{2+}$ release supports the presence of continuous electrical connectivity of ATs with the surface sarcolemma, presumably via sparse TTs as a conduit for ECC activation.

Human left atrial myocardium exhibits axial RyR2 cluster morphologies at ATs. To assess whether AT membrane structures are associated with junctional RyR2 clusters in human tissue as well, we developed dual-color immunolabeling protocols for STED nanoscopy of left atrial (LA) tissue sections. Cav3-labeled sections showed highly ordered rod-shaped AMs aligned with capillaries in atrial tissue (Figure 2A). Interestingly, AMs with robust intracellular Cav3 staining showed that multiple central ATs regularly intersected with transversal RyR2 cluster striations (Figure 2A), similar to our observations in isolated human and mouse AMs (Figure 1G). Importantly, higher magnifications inside tissue-embedded AMs showed distinct, axially extended RyR2 cluster morphologies, which tightly embraced the Cav3-labeled ATs (Figure 2B). Thus, central AT-associated (but not off-membrane CY) RyR2 clusters revealed a distinct morphology in human LA sections, in keeping with the possibility of unique axial couplon functions such as for the rapid $\mathrm{Ca}^{2+}$ release onset observed in murine AMs.

Differential regulation of atrial RyR2 clusters. Our finding that local $\mathrm{Ca}^{2+}$ release occurs earlier at AT sites suggests potentially unknown local regulatory mechanisms. In VMs, TT membranes were previously shown to contain $\beta$-adrenergic receptor ( $\beta$-AR) and adenylyl cyclase (AC5/6) signaling structures, increasing cAMP concentrations locally $(26,27)$. Furthermore, cAMP sensing through PKA is directly tethered to each subunit of the homotetra- 
A

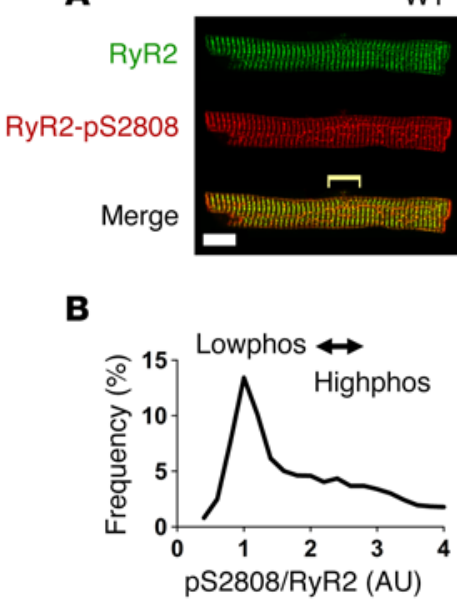

WT

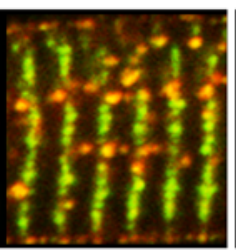

RyR2-S2808A $\mathrm{A}^{+/ 4}$

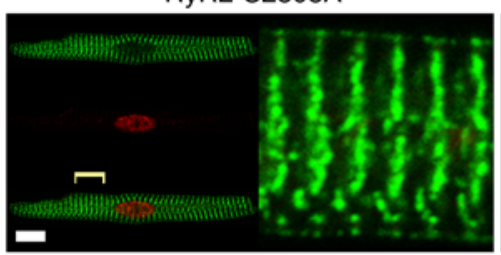

$\mathrm{WT}+\mathrm{H} 89$

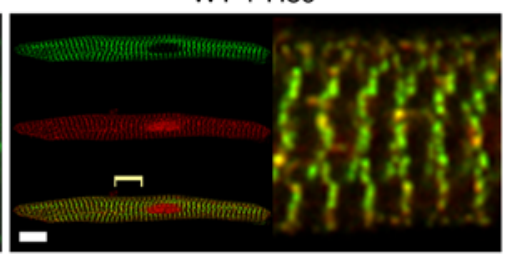

C

RyR2-pS2808

Merge
WT

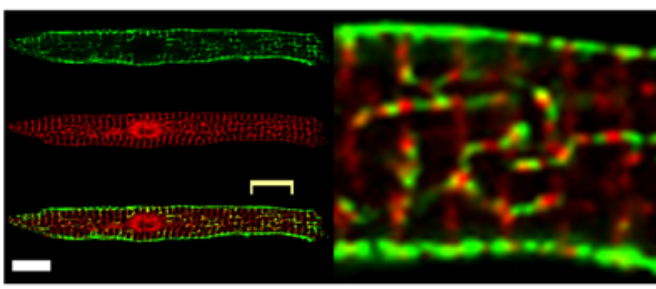

D Cav3 highphos

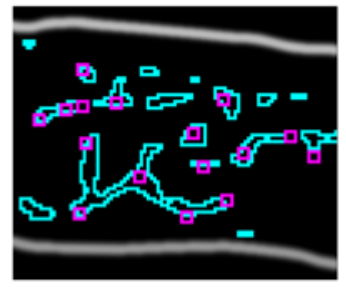

E

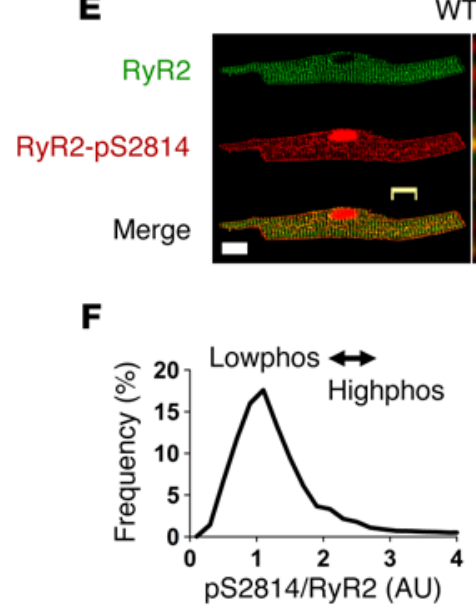

RyR2-S2814A $\mathrm{A}^{+/+}$

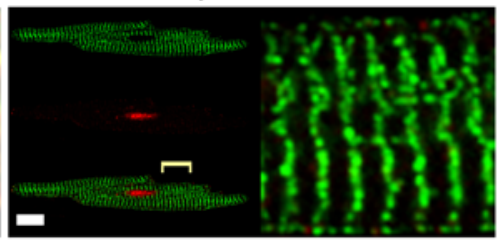

G

RyR2-pS2814

Merge
WT

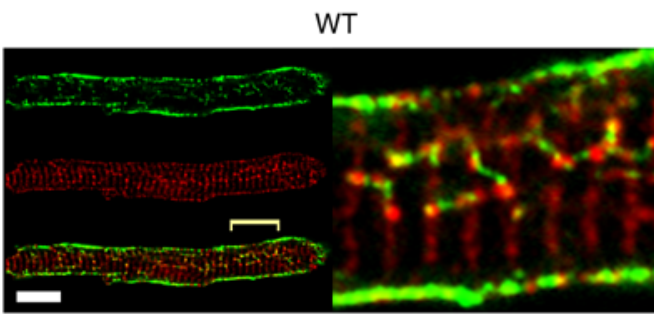

H

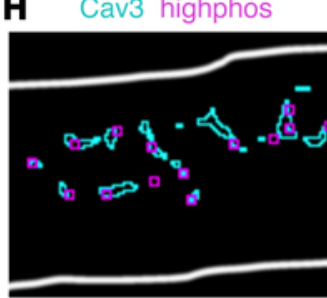

\section{I}

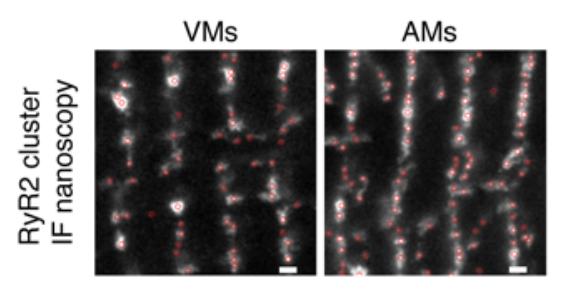

J

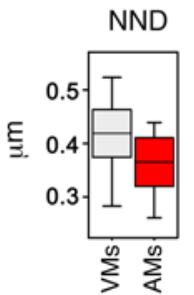

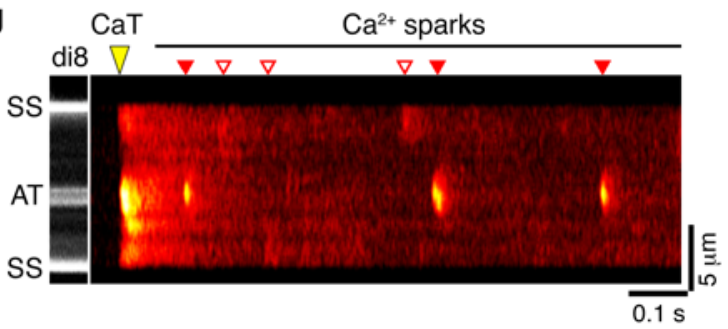

Figure 3. Differential regulation of RyR2 cluster phosphorylation. (A) AMs coimmunostained for RyR2- and PKA-phosphorylated RyR2-pS2808. WT: highphos RyR2 clusters (yellow) aligned in central-axial string-of-pearls, intersecting transverse striations of lowphos RyR2 clusters (green); RyR2-

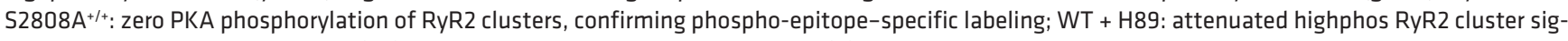
nals. Scale bars: $10 \mu \mathrm{m}$; magnified ( $\times 4$ ) regions are indicated by yellow brackets. (B) Histogram showing bimodal frequency distribution of in situ pS2808/ RyR2 normalized cluster signals in untreated WT AMs. The main peak indicates abundant lowphos versus fewer highphos RyR2 clusters represented by the shoulder. $n=22$ AMs. (C) AMs colabeled for Cav3 and RyR2-pS2808. Highphos RyR2-pS2808 clusters alternated with Cav3 clusters in central AT structures. Scale bar: $10 \mu \mathrm{m}$; magnification $\times 4$. (D) Image segmentation confirming highphos RyR2-pS2808 clusters aligned with Cav3-labeled TAT structures. (E) WT, RyR2-S2814A ${ }^{+/+}$, and WT AMs treated with the CaMK inhibitor AIP were coimmunostained for RyR2 and the CaMK-phosphorylated epitope RyR2pS2814. Note the central highphos RyR2 clusters (yellow) versus lowphos RyR2 clusters (green). AMs from CaMK phosphorylation-incompetent RyR2-

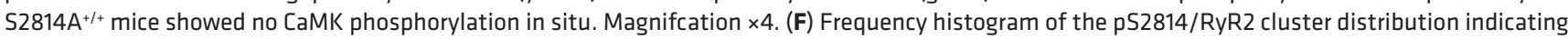
abundant lowphos versus few highphos RyR2 clusters in control AMs. $n=19$ AMs. (G) AMs coimmunostained for Cav3 and RyR2-pS2814. Highphos RyR2 clusters were associated with Cav3-labeled AT structures as confirmed by image segmentation (H). Scale bar: $10 \mu \mathrm{m}$, magnifcation $\times 4$. (I) STED images comparing immunolabeled central RyR2 clusters in VMs versus AMs. Atrial RyR2 clusters identified by red circles show a higher density on Z-lines and a trend toward overall shorter NNDs. Images are representative of $18 \mathrm{VMs}$ and 19 AMs. Scale bars: $500 \mathrm{~nm}$. Box and whisker plot: boxes show lower and upper quartiles; whiskers represent the $5^{\text {th }}$ and $95^{\text {th }}$ percentiles. IF, immunofluorescence. (J) Confocal live AM imaging with di-8-ANEPPS and fluo-4 showing large AT-localized $\mathrm{Ca}^{2+}$ sparks occurring repeatedly. CaT, final 1-Hz pacing evoked $\mathrm{Ca}^{2+}$ transient; red triangles indicate AT-associated $\mathrm{Ca}^{2+}$ sparks; white triangles indicate surface membrane-associated $\mathrm{Ca}^{2+}$ sparks. 
meric RyR2 channel complex (28). Since PKA phosphorylation of Ser 2808 and CaMK phosphorylation of Ser 2814 are powerful positive regulatory mechanisms of RyR2 sensitivity for $\mathrm{Ca}^{2+}$-dependent activation (28-30), we investigated the nature of subcellular RyR2 phosphorylation using novel in situ immunofluorescence strategies.

Using Abs specific to either PKA-phosphorylated RyR2pS2808 or CaMK-phosphorylated RyR2-pS2814, we identified distinct populations of highly phosphorylated (highphos) RyR2 clusters in AMs (Supplemental Figure 5A), but not in VMs from the same WT mouse hearts (Supplemental Figure 5B). In AMs, central highphos RyR2-pS2808 clusters were axially aligned in "stringof-pearls" arrangements, which regularly intersected with minimally phosphorylated (lowphos) clusters in transversal striations at Z-lines (Figure 3A). Molecular labeling specificity was confirmed by 2 independent approaches. First, RyR2-pS2808 epitope detection was obstructed by the blocking, but not by the control peptide, confirming specific phospho-Ab binding (Supplemental Figure 6). Second, AMs from mice expressing a PKA phosphorylation-incompetent mutation ( $R y r 2^{S 2808 A / S 2808 A}$; hereafter referred to as RyR2-S2808A ${ }^{+/+}$-knockin mice) showed complete ablation of RyR2-pS2808 in situ cluster signals (Figure 3A).

Since local PKA activity, apparent through highphos RyR2 cluster phosphorylation, occurred in the absence of $\beta$-AR stimulation, we hypothesized that competitive pharmacological PKA inhibition would attenuate TAT-associated PKA activity. Indeed, live AMs treated with the cAMP-specific PKA inhibitor H89 (50 $\mu \mathrm{M})$ showed blunted highphos RyR2 cluster signals (Figure 3A). This result indicates significant cAMP-dependent local PKA activity, sufficient to sustain PKA phosphorylation of highphos RyR2 clusters. Collectively, these data suggest a highly compartmentalized form of atrial RyR2 cluster regulation, which may functionally differentiate highphos from lowphos RyR2 channel populations.

To quantify the apparently heterogeneous in situ cluster phosphorylation, we analyzed the frequency distribution of the pS2808/RyR2 normalized cluster signals. Untreated WT AMs showed a major pS2808/RyR2 peak, indicating abundant lowphos clusters (Figure 3B). In contrast, highphos pS2808/RyR2 signals occurred at much lower frequencies, indicated by a rightskewed distribution (Figure 3B). VMs isolated from the same hearts showed a markedly different distribution (Supplemental Figure 5C). To explore whether axially aligned RyR2 highphos clusters are associated with AT structures in AMs, we coimmunostained AMs for Cav3 and RyR2-pS2808. This confirmed that Cav3-positive ATs were closely aligned with highphos RyR2 signals in string-of-pearls arrangements (Figure 3C). Furthermore, triple staining of Cav3, RyR2, and RyR2-pS2808 confirmed that RyR2 clusters that colocalized with Cav3-labeled AT structures were indeed highphos clusters (Supplemental Figure 7). In contrast, lowphos RyR2 clusters in transverse striations did not colocalize with AT or TT components.

Using a corresponding image segmentation strategy, we analyzed the spatial association of highphos versus lowphos RyR2pS2808 clusters with Cav3-labeled TAT structures as the nearest neighbor distance (NND) (Supplemental Figure 8A). The NND was significantly smaller for highphos than for lowphos RyR2pS2808 clusters (Supplemental Figure 8B). We visualized the segmentation analysis output and compared corresponding image regions and found that central highphos RyR2-pS2808 clusters were tightly associated with Cav3-positive AT structures (Figure 3D). While highphos RyR2-pS2808 clusters represented the smaller population in untreated AMs (Figure 3B), our analysis indicated their preferential association with AT structures, consistent with rapid junctional $\mathrm{Ca}^{2+}$ release at $\mathrm{AT}$ sites.

In addition, CaMK phosphorylation of the RyR2-S2814 site was identified as a mechanism of AF susceptibility in mice (31, 32), and both RyR2-S2808 and RyR2-S2814 phosphorylation changes were identified in atrial samples from patients with $\mathrm{AF}$ $(33,34)$. Consequently, we investigated RyR2-pS2814 phosphorylation in situ, finding axially aligned highphos clusters (Figure 3E). Confirming phospho-epitope-specific labeling, AMs from CaMK phosphorylation-incompetent Ryr2 $2^{\text {S2814/S2814A }}$-knockin mice (referred to herein as RyR2-S2814A ${ }^{+/+}$-knockin mice) showed complete ablation of RyR2-pS2814 cluster signals (Figure 3E). Furthermore, pretreatment with the CaMK-specific autoinhibitory peptide (AIP, $5 \mu \mathrm{M}$ ) blunted the RyR2-pS2814 highphos RyR2 cluster signal in situ (Figure 3E).

The pS2814-RyR2 frequency histogram showed a major peak and right-skewed distribution, indicating abundant lowphos and few highphos clusters (Figure 3F). Coimmunostaining of AMs for Cav3 and RyR2-pS2814 showed that highphos RyR2-pS2814 clusters aligned with AT structures (Figure 3G). While image segmentation of Cav3-labeled TAT structures and highphos RyR2-pS2814 clusters confirmed this association, some highphos clusters showed no TAT association (Figure $3 \mathrm{H}$ ). And while statistical analysis of RyR2-pS2814 to Cav3 spacing confirmed a specific correlation (Supplemental Figure 8C), NND analysis did not reach statistical significance between highphos and lowphos RyR2-pS2814 clusters using analogous thresholding procedures applied to RyR2-pS2808 clusters (Supplemental Figure 8D). Taken together, our data on untreated AMs suggest that locally increased CaMK activity can maintain RyR2-S2814 cluster phosphorylation at both TAT structures and other sites. Thus, both CaMK and PKA phosphorylate AT-associated RyR2 clusters in AMs, albeit through distinct molecular pathways converging locally in highphos clusters.

Large elemental $\mathrm{Ca}^{2+}$ signals at AT sites. Local control of elemental $\mathrm{Ca}^{2+}$ release $\left(\mathrm{Ca}^{2+}\right.$ sparks) in CRUs may depend on the spatial interrelation between RyR2 clusters and AT membranes, as proposed previously (7). Using STED nanoscopy, we compared intracellular RyR2 cluster densities between AMs and VMs (Figure 3I). Interestingly, there was a nonsignificant trend (Mann-Whitney $U$ test) toward a smaller yet highly variable NND between RyR2 clusters in AMs, suggesting a potentially higher central cluster density (Figure 3I). Since AT structures are significantly larger in AMs, their membrane surface may couple more and/or larger RyR2 clusters during local $\mathrm{Ca}^{2+}$ release than do TTs, as supported by ET and 3D segmentation (Figures 1, D and E).

To explore this hypothesis, we imaged fluo-4-loaded AMs costained with di-8-ANEPPS to determine whether $\mathrm{Ca}^{2+}$ sparks originate from distinct RyR2 cluster sites. Transversal line scanning during 1-Hz field pacing confirmed earlier central $\mathrm{Ca}^{2+}$ transient onset (analogous to Figure $1 \mathrm{~K}$ ), followed by unusually large post-pacing $\mathrm{Ca}^{2+}$ macro-sparks fired repeatedly from the same AT site (Figure 3J). Apparently, $\mathrm{Ca}^{2+}$ sparks originating at the surface membrane were smaller. Taken together, CRUs at AT structures 
A
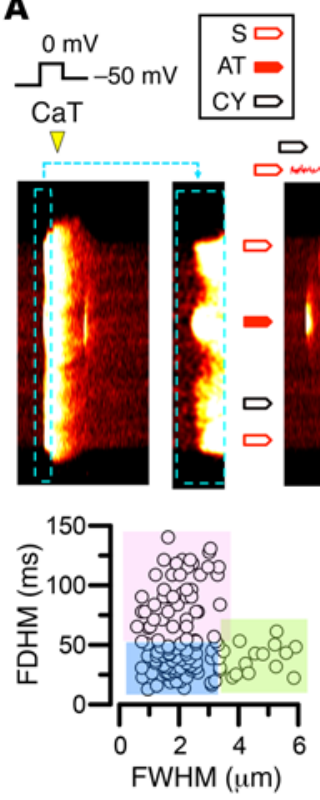

단.

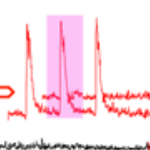

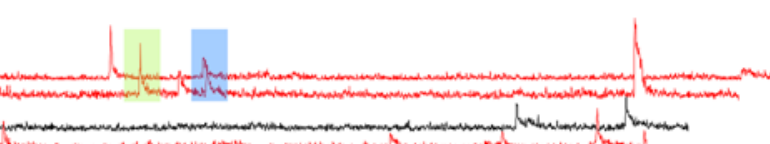
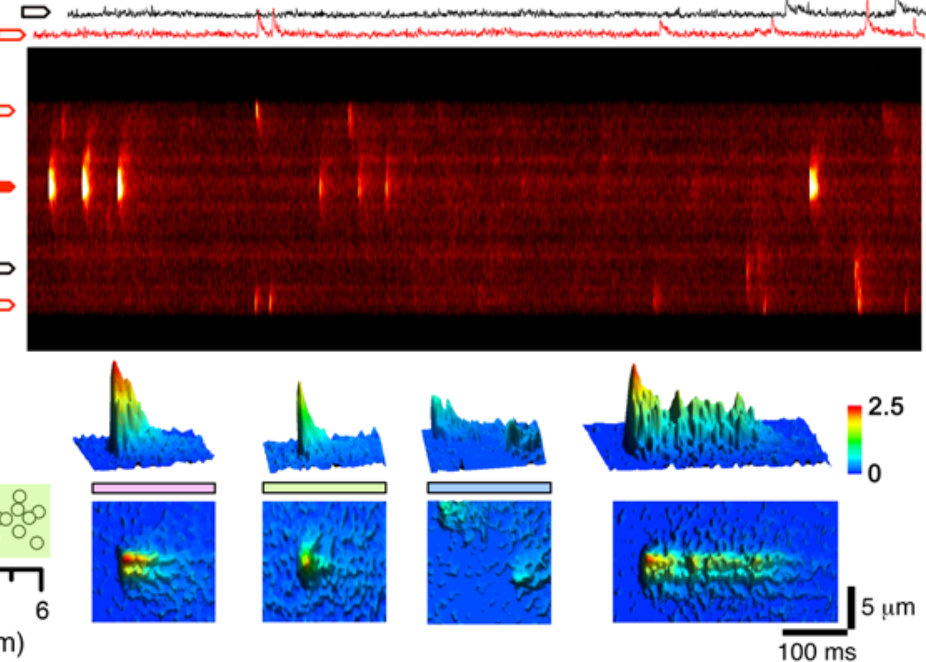

\section{C}

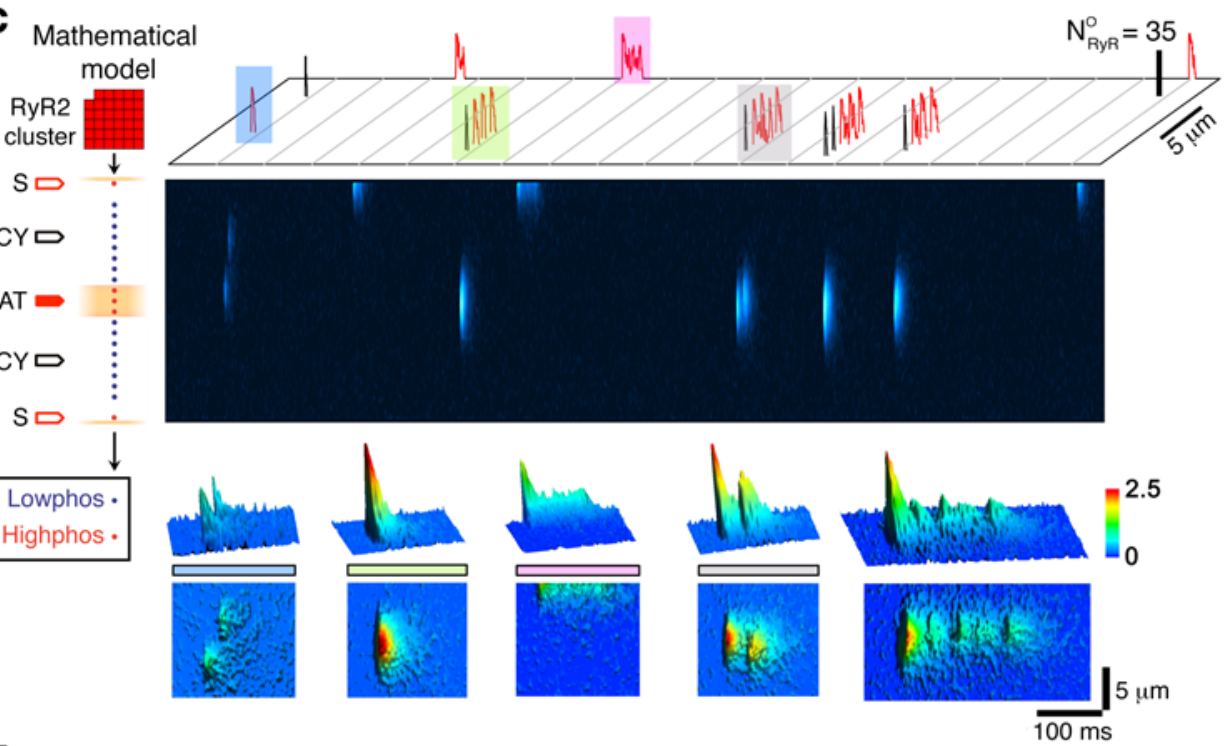

B
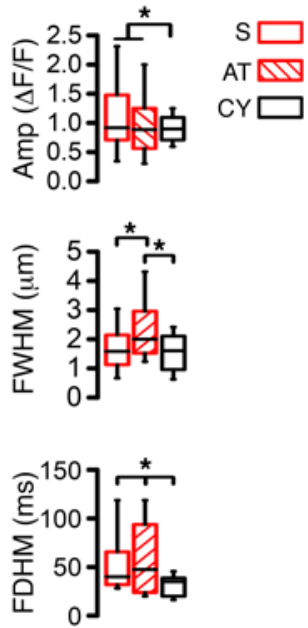

D
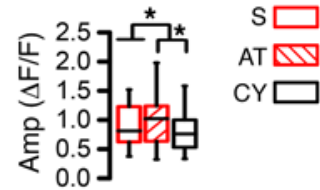

E

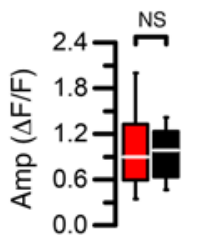

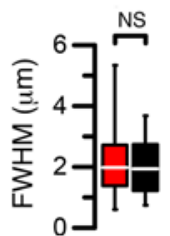

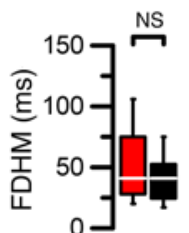

AM imaging (from B)

Spark modeling (from D)

Figure 4. $\mathrm{Ca}^{2+}$ macro-sparks at AT sites are reproduced by RyR2 cluster modeling. (A) The final of 5 consecutive $\mathrm{Ca}^{2+}$ transients of a voltage-clamped $\mathrm{AM}$ (1- $\mathrm{Hz}$ pacing) during depolarization from -75 to $0 \mathrm{mV}$ was magnified (corresponding dashed-line boxes) to visualize early $\mathrm{Ca}^{2+}$ signal onset locally. Block arrows indicate different $\mathrm{Ca}^{2+}$ spark locations at $-50 \mathrm{mV}$, including off-membrane cytosolic sites (CY). Top 2D traces show $\mathrm{Ca}^{2+}$ spark events at AT, S, and CY locations, and examples highlighted by color are magnified by corresponding 3D surface plots (bottom panels): macro-spark (pink), ember (green), classic $\mathrm{Ca}^{2+}$ sparks (blue), and regenerative macro-sparks. Dot plot correlating $\mathrm{Ca}^{2+}$ spark full duration at half maximum (FDHM) and full width at half maximum (FWHM) to segregate classic (blue) from macro-sparks (green) and embers (pink). (B) Box plots comparing Ca ${ }^{2+}$ spark amplitude (Amp), FWHM, and FDHM in different locations. ${ }^{*} P<0.05$, by ANOVA. (C) Atrial $\mathrm{Ca}^{2+}$ spark model composed of 1 transversal RyR2 cluster row. The indicated AT and $\mathrm{S}$ structures were associated with junctional highphos RyR2 clusters. Each cluster contained 35 RyR2 channels stochastically activated at $-50 \mathrm{mV}$; both highphos and lowphos RyR2 clusters initiated SR Ca+ ${ }^{2+}$ release. Mathematical modeling generated the stochastic open time profiles of individual RyR2 channels for each cluster, which was used to calculate line-scan images of $\mathrm{Ca}^{2+}$ sparks with confocal resolution. $\mathrm{Ca}^{2+}$ sparks presented as $2 \mathrm{D}$-flattened images (top) are highlighted by color, and the corresponding 3D surface plots are shown (bottom): classic Ca ${ }^{2+}$ sparks (blue), macro-spark (green), ember (pink), and regenerative macro-sparks (gray). $\mathrm{N}_{\text {RyR }}^{0}$, number of open RyR2 channels. (D) Box and whisker plots comparing Ca ${ }^{2+}$ spark amplitude, FWHM, and FDHM as the average modeling output. (E) Comparing the aggregate properties of calcium sparks show no significant differences between the AM imaging results and spark modeling output. ${ }^{*} P<0.05$, by ANOVA (B and $\mathbf{D}$ ). Box and whisker plots: boxes show lower and upper quartile; whiskers show the 5 th and 95 th percentiles. 
A

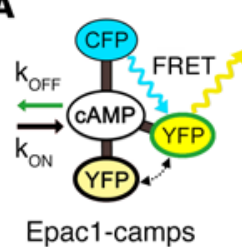

FRET regions AM

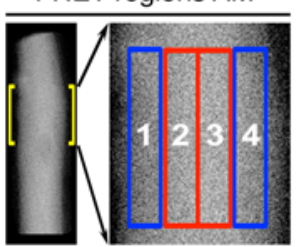

B

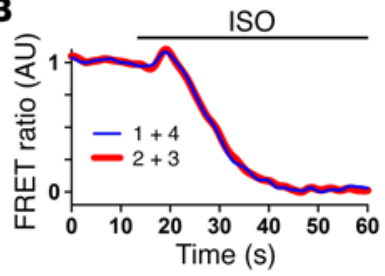

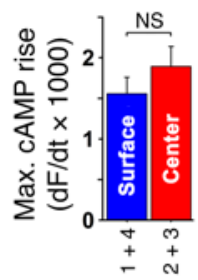

C

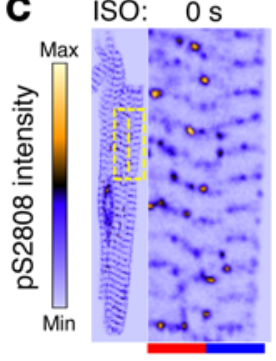

$10 \mathrm{~s}$

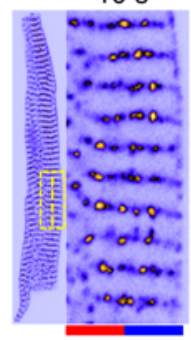

$20 \mathrm{~s}$

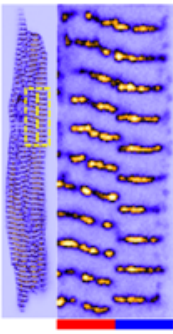

$60 \mathrm{~s}$

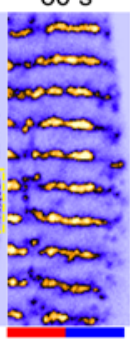

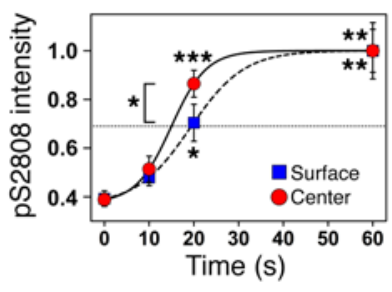

D

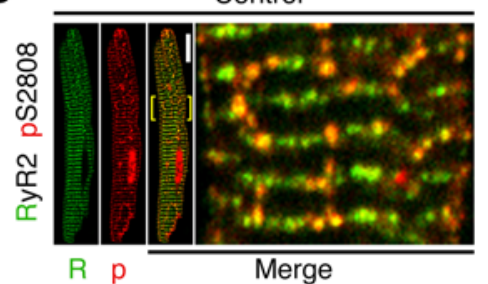

E

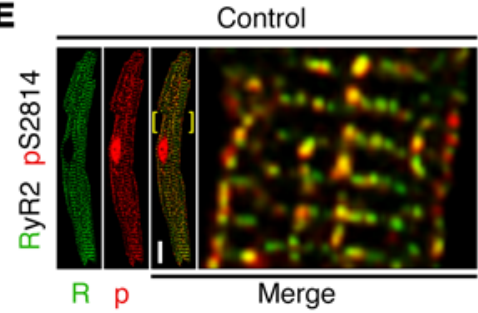

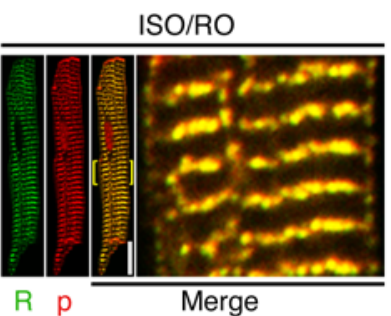

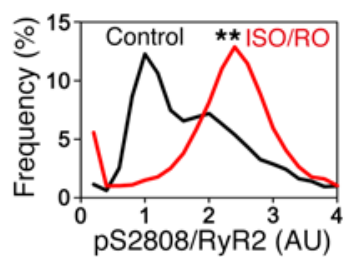

ISO/RO

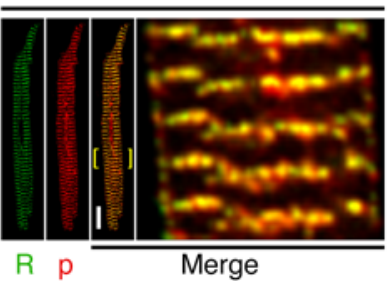

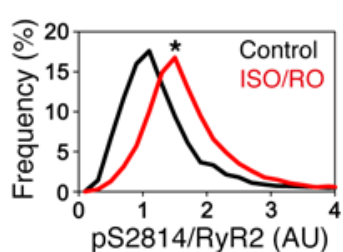

Figure 5. In situ regulation of atrial RyR2 cluster phosphorylation. (A) Illustration showing cAMP-dependent responses of Epac1-camps, a ubiquitous cytosolic FRET sensor, during live-cell measurements. In AMs, signals from 4 transversally distributed FRET regions were sampled and signal averaged pairwise for corresponding central $\left(2+3\right.$, red) versus surface $\left(1+4\right.$, blue) regions. $\mathrm{k}_{\mathrm{OFF}}$, CAMP dissociation constant; $\mathrm{k}_{\mathrm{ON}}$, CAMP association constant; CFP, cyan fluorescent protein; YFP, yellow fluorescent protein. (B) FRET ratio traces show the same spatiotemporal AM response to $0.1 \mu \mathrm{M}$ ISO stimulation during increasing intracellular CAMP generation. Bar graph comparing the maximal rate of cAMP rise shows no significant difference between AM center and surface. NS, by Student's $t$ test. $n=12$ AMs. (C) Confocal time series showing progressive RyR2-S2808 cluster phosphorylation following up to 60 seconds of $\beta$-adrenergic stimulation (1 $\mu$ M ISO). RyR2-pS2808 cluster signals were averaged from 10 striations (see magnifications) through equal-sized central (red) and surface (blue) regions indicated by dashed-line yellow boxes. RyR2-pS2808 intensity plot shows progressive cluster phosphorylation, which was significantly faster for central AM regions after a 20 -second ISO stimulation. Each time point represents 7 AM experiments. ${ }^{*} P<0.05$,

${ }^{* *} P<0.01$, and ${ }^{* *} P<0.001$, by ANOVA. ( $\mathbf{D}$ and $\left.\mathbf{E}\right)$ Maximal stimulation by combined ISO/RO $(1 \mu \mathrm{M} / 10 \mu \mathrm{M})$ treatment converted lowphos into highphos RyR2 clusters. (D) Histogram shows bimodal pS2808-RyR2 cluster signal pattern under control conditions (black trace), resulting in a profound change of the lowphos RyR2 cluster signals by ISO/RO treatment: 1 major highphos peak, consistent with complete lowphos cluster conversion (red trace).

${ }^{* *} P<0.01$, by Mann-Whitney $U$ test. Dara are representative of 13 control-treated and 18 ISO/RO-treated AMs. Scale bars: $10 \mu \mathrm{M}$, magnification $\times 4$. (E) Images and histogram showing a distinct pS2814/RyR2 cluster phosphorylation shift after ISO/RO stimulation (note: control data are the same as in Figure 3F). ${ }^{*} P<0.05$, by Mann-Whitney $U$ test. Data are representative of 19 control-treated and 12 ISO/RO-treated AMs. Scale bars: $10 \mu M$, magnification $\times 4$.

can generate $\mathrm{Ca}^{2+}$ macro-sparks, suggesting a potential mechanism of local AT-dependent $\mathrm{Ca}^{2+}$ signaling in AMs.

To control LCC activity, voltage-clamped AMs underwent 5 depolarization cycles to equilibrate the $\mathrm{SR} \mathrm{Ca}^{2+}$ load, followed by imaging of spontaneously occurring $\mathrm{Ca}^{2+}$ sparks. We used $\mathrm{dF} / \mathrm{dt}$ onset analysis to identify early $\mathrm{Ca}^{2+}$ release sites (as shown in Figure $1 \mathrm{M}$ ) and classified the principal $\mathrm{Ca}^{2+}$ spark locations accordingly. Following repolarization to $-50 \mathrm{mV}, \mathrm{Ca}^{2+}$ macro-sparks, characterized by high amplitude, large width, and long duration, originated repeatedly from AT sites (Figure $4 \mathrm{~A}$ ). In contrast, $\mathrm{Ca}^{2+}$ sparks originating from non-AT-coupled sites appeared smaller (Figure 4A). 2D traces and 3D surface plots highlight the different types of $\mathrm{Ca}^{2+}$ spark events: macro-sparks (pink), long-lasting sparks (embers; green), classic sparks (blue), and regenerative macro-spark $\mathrm{Ca}^{2+}$ propagation (Figure 4A, compare color-rendered top and bottom panels). Central $\mathrm{Ca}^{2+}$ macro-sparks, apparently sequentially recruited, indicated that several RyR2 clusters may contribute to the same $\mathrm{Ca}^{2+}$ release event. The dot plot summarizes the differ- 
ent types (grouped by colors, as above) of $\mathrm{Ca}^{2+}$ signals originating from AT sites: classic sparks, macro-sparks, and embers (Figure $4 \mathrm{~A})$. Comparison of the mean width and duration at different subcellular locations showed that $\mathrm{Ca}^{2+}$ macro-sparks originated more frequently from ATs (Figure 4B). This suggests that AT junctions can repeatedly generate $\mathrm{Ca}^{2+}$ macro-sparks and embers, generally coordinating larger local $\mathrm{Ca}^{2+}$ release events.

Quantitative RyR2 cluster modeling supports local and global AT coupling. To integrate the observed local and global $\mathrm{Ca}^{2+}$ signals mechanistically, we used an existing mathematical ECC model that incorporates the latest biophysical information regarding local RyR2 cluster dynamics (35). This model was modified to account for our data on AT-associated $\mathrm{Ca}^{2+}$-signaling functions and discrete localizations of highphos RyR2 clusters at AT and $\mathrm{S}$ sites (Figure $4 \mathrm{C}$ ). We built a 3D model of CICR within a single AM sarcomeric Z-region (M-line to M-line) containing CRUs with either lowphos or highphos RyR2 clusters ( $n=35$ RyR2 channels each), randomly arranged transversally to simulate AT-associated $\mathrm{Ca}^{2+}$ signals recorded in line-scan mode. RyR2 clusters, spontaneously activated by the local diadic subspace $\left[\mathrm{Ca}^{2+}\right]_{\mathrm{ds}}$ concentration or by LCC openings at the $-50 \mathrm{mV}$ holding potential, produced corresponding $\mathrm{Ca}^{2+}$ sparks and localized depletions in junctional SR Ca ${ }^{2+}$ concentration $\left(\left[\mathrm{Ca}^{2+}\right]_{\mathrm{iSR}}\right.$ ) termed $\mathrm{Ca}^{2+}$ blinks (Supplemental Figure 9). Detailed visualization of the simulated $\mathrm{Ca}^{2+}$ spark behaviors and their spatiotemporal dimensions showed that $\mathrm{Ca}^{2+}$ macro-sparks and embers could be elicited in the model from AT highphos RyR2 clusters (Figure 4C). Systematic $\mathrm{Ca}^{2+}$ spark comparisons showed a significantly increased $\mathrm{Ca}^{2+}$ spark amplitude at $\mathrm{AT}$ sites and reproduced $\mathrm{Ca}^{2+}$ spark dimensions distinct from those at cytosolic and subsarcolemmal sites (Figure 4D). Average $\mathrm{Ca}^{2+}$ spark dimensions were quantitatively reproduced by computational modeling (Figure 4E). Thus, mathematical modeling supports the plausibility of a new $\mathrm{Ca}^{2+}$-signaling mode, whereby AT-associated structures constitutively regulate atrial ECC through highphos RyR2 clusters, locally increasing the sensitivity for $\mathrm{Ca}^{2+}$-dependent activation as a basis for $\mathrm{Ca}^{2+}$ macro-spark generation and local $\mathrm{Ca}^{2+}$ transient onset.

$\beta$-AR stimulation recruits nonjunctional RyR2 clusters. Lowphos RyR 2 clusters might be selectively recruited during $\beta$-AR stimulation, as opposed to highphos clusters constantly saturated by tonic local PKA and CaMK phosphorylation. To characterize intracellular cAMP concentration changes in real time, we used transgenic mice that express a ubiquitous cytosolic fluorescence resonance energy transfer (FRET) sensor, Epac1-camps (36) (Figure 5A). Pairwise regional FRET sampling in AMs during isoproterenol (ISO) stimulation was used to detect central versus peripheral cAMP concentration changes (Figure 5A). The resulting traces, each from central and peripheral regions, showed comparable robust FRET signal responses as judged by the maximal amplitude of the intracellular cAMP rise after approximately 20-second ISO stimulation (Figure 5B). Also, the maximal rate of the cAMP rise $(\mathrm{dF} / \mathrm{dt})$ was not different between central or peripheral AM regions (Figure 5B). Given that cytosolic cAMP movement, at least in VMs, is much slower than free diffusion (37) and that principally similar cAMP concentration changes were present in the center and periphery of AMs, our data support a central, potentially AT-associated cAMP-generating mechanism.
To assess central cAMP-dependent PKA signaling in AMs, we studied the spatiotemporal dynamics of lowphos RyR2 cluster recruitment during acute ISO stimulation $(1 \mu \mathrm{M})$. We analyzed RyR2-pS2808 signal intensities on RyR2 cluster striations, comparing central versus surface regions for temporal differences (Figure 5C). RyR2-S2808 cluster phosphorylation on transversal cluster striations was significantly higher 20 seconds after ISO stimulation (Figure 5C), similar to the observed cAMP FRET signal dynamics. This supports the notion of faster and/or more efficient central cAMP-dependent signal transduction inside AMs (Figure 5C).

To maximally recruit RyR2 clusters, we combined $\beta$-AR stimulation (1 $\mu \mathrm{M}$ ISO) with selective phosphodiesterase PDE4D inhibition by $10 \mu \mathrm{M}$ rolipram (RO). This combined ISO/RO treatment converted all lowphos into highphos RyR2-pS2808 clusters, in contrast to control AMs treated with carrier solution (Figure 5D). Consistent with maximal lowphos RyR2 cluster conversion, ISO/ RO treatment significantly transformed the pS2808/RyR2 cluster population signal from a lowphos-dominated peak into a single highphos cluster peak (Figure 5D). In addition, ISO/RO treatment shifted the pS2814/RyR2 signal peak rightward (Figure 5E). Apparently, the peak RyR2/pS2808 highphos cluster conversion following ISO/RO stimulation is more pronounced. These data confirm that nonjunctional RyR2 cluster regulation in situ can be explained by bimodal cluster behavior through reversible catecholaminergic recruitment of the overall larger lowphos RyR2 cluster population, through both increased PKA and CaMK phosphorylation.

Since $\beta$-AR stimulation is a central inotropic, yet incompletely understood, mechanism in the atria, we studied intracellular $\mathrm{Ca}^{2+}$ transient behavior during $0.5-\mathrm{Hz}$ pacing. AMs with abundant AT structures reproduced the early $\mathrm{Ca}^{2+}$ release profile led by AT site activation under control conditions (Figure 6A). ISO/RO-treated cells showed profoundly accelerated $\mathrm{Ca}^{2+}$ transients with flattened profiles, indicating more even activation of junctional and nonjunctional (CY) $\mathrm{Ca}^{2+}$ release sites (Figure 6A). While the earliest $\mathrm{Ca}^{2+}$ release sites were stably associated with either AT or S locations (shown by di-8-ANEPPS staining), the mean $\mathrm{Ca}^{2+}$ release onset was significantly accelerated at $\mathrm{S}$, but not at AT, locations (Figure 6A). Thus, analysis of different $\mathrm{Ca}^{2+}$ release locations by derivative $(\mathrm{dF} / \mathrm{dt})$ showed that the main ISO/RO effect consists of accelerated cytosolic $\mathrm{Ca}^{2+}$ release (Figure 6A), compatible with nonjunctional lowphos RyR2 cluster recruitment leading to accelerated $\mathrm{Ca}^{2+}$ release spread during cAMP-dependent stimulation.

In VMs, cAMP is generated by transmembrane AC5/6, and $\beta$-AR stimulation represents the major pathway to increase AC5/6 activity (27). However, this has not been established in atrial muscle. We confirmed similar AC5/6 protein expression levels in murine atrial and ventricular tissue (Figure 6B). Interestingly, expression of the PKA catalytic subunit (PKA-C) was significantly higher in the atria (Figure 6B and Supplemental Figure 10), correlating with more robust PKA-C striations in AMs compared with those observed in VMs (Supplemental Figure 11). Since RyR2 channels and PKA-C were shown to colocalize in situ at Z-line striations (based on previous cAMP FRET measurements; ref. 28), and since the central RyR2 cluster density may be higher in AMs (Figure 3I), our data suggest an increased atrial capacity for cAMP-dependent regulation of RyR2 clusters. Indeed, immunoblots confirmed that the maximal change of substrate-normalized 
A
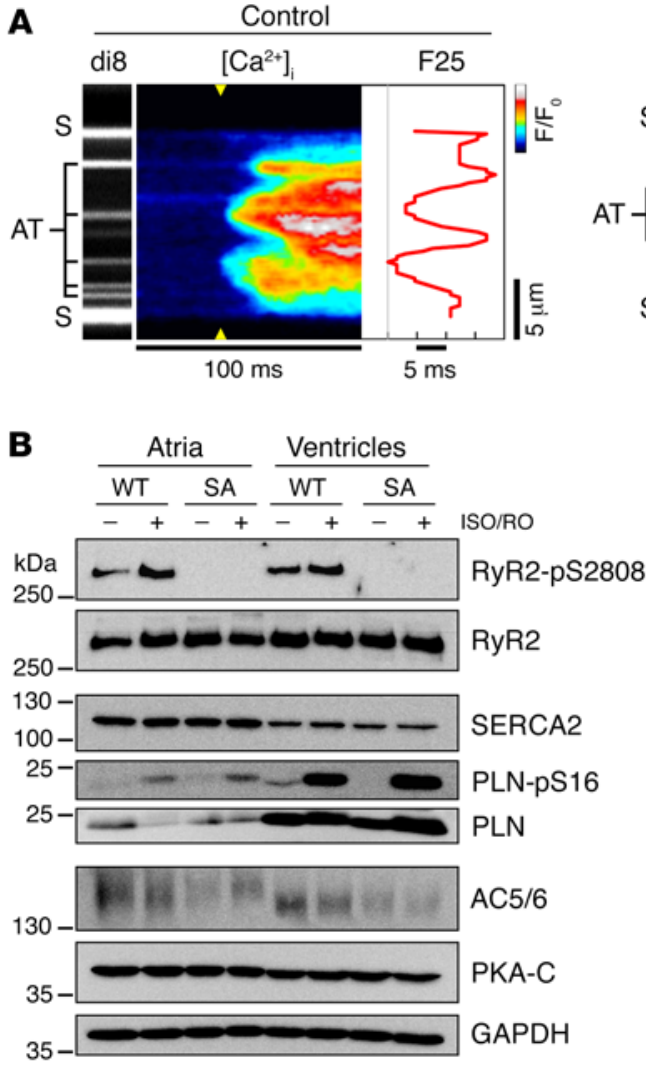

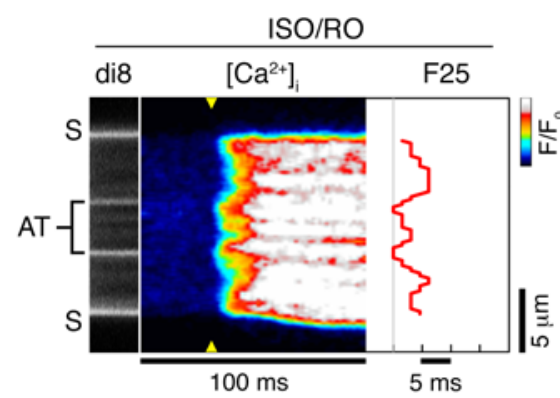

WT
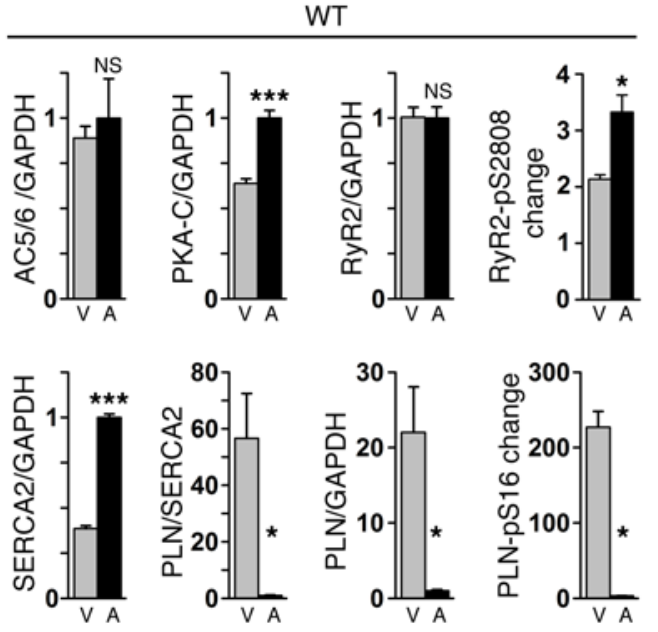

C

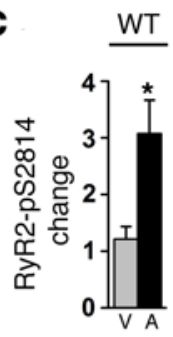

D

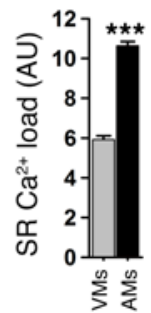

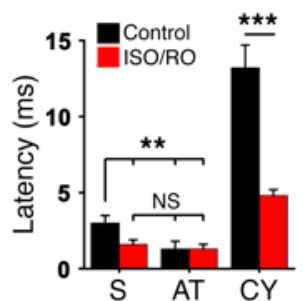

Figure 6. Catecholaminergic stimulation accelerates atrial $\mathrm{Ca}^{2+}$ signaling. (A) Live imaging of AT and $\mathrm{S}$ membrane structures during $\mathrm{Ca}^{2+}$ transient onset in control- versus ISO/RO-treated AMs (0.5- $\mathrm{Hz}$ pacing). ISO/RO treatment accelerated transversal $\mathrm{Ca}^{2+}$ release propagation across the cytosol, compressing the F25 profile through a decreased cytosolic (CY) latency of early $\mathrm{Ca}^{2+}$ release (dF/dt). Data are representative of 9 control-and 17 ISO/RO-treated AMs. ${ }^{* *} P<0.01$ and ${ }^{* * *} P<0.001$, by Student's $t$ test. (B) Immunoblots comparing ISO/RO-treated $(1 \mu \mathrm{M} / 10 \mu \mathrm{M})$ atrial (A) tissue from WT and phosphorylation-incompetent RyR2-S2808 ${ }^{+/+}$mouse hearts; ventricular (V) tissue was compared for reference. In WT atria, significantly higher expression levels of both PKA-C and SERCA2 were detected, in contrast to significantly lower PLN levels. Atrial versus ventricular PKA phosphorylation changes were significantly increased for the RyR2-S2808 site, but not for the PLN-S16 site. Note the similar AC5/6 and RyR2 protein levels. Blots are representative of 3 individual experiments (see Supplemental Figure 10 for uncut blots). (C) Analogous RyR2-S2814-specific immunoblotting established in CaMK phosphorylation-incompetent RyR2-S2814A $\mathrm{A}^{++}$mouse hearts (see also Supplemental Figures 12A and 12B). Bar graph shows a significantly increased atrial RyR2-S2814 phosphorylation change in atrial tissue after ISO/RO $(1 \mu \mathrm{M} / 10 \mu \mathrm{M})$ treatment. (D) SR Ca ${ }^{2+}$ load was significantly higher in AMs than in VMs measured with caffeine $(10 \mathrm{mM}) . n=8 \mathrm{AMs}, 9$ VMs. ${ }^{*} P<0.05$ and ${ }^{* *} P<0.001$, by Student's $t$ test.

RyR2-S2808 phosphorylation by PKA (Figure 6B) and RyR2-S2814 by CaMK (Figure 6C and Supplemental Figure 12, A and B) during ISO/RO treatment was significantly higher in AMs compared with that detected in VMs. In contrast, acute pharmacological ISO stimulation using a low nanomolar concentration $(20 \mathrm{~nm})$ resulted in a significant change in RyR2-S2808 phosphorylation, but no change in RyR2-S2814 phosphorylation (Supplemental Figure 12C). Taken together, our data indicate that synergistic regulation of lowphos RyR2 clusters by both PKA and CaMK occurs during maximal sustained cAMP generation through a highly efficient regulatory mechanism in AMs, with the potential for complete lowphos-to-highphos RyR2 cluster conversion.

Earlier data from rat hearts showed that phospholamban (PLN), an inhibitor of SR $\mathrm{Ca}^{2+}$ (SERCA2) pump function released by PKA phosphorylation of PLN-S16, is expressed at significantly lower levels in AMs than in VMs (16). In addition, SERCA2 expression is approximately 2-fold higher in AMs compared with expression in VMs, while the PLN/SERCA2 ratio is reduced by approximately 4 -fold, suggesting decreased stoichiometric SERCA2 inhibition in atria $(12,16)$. We confirmed significantly higher SERCA2 levels, but since PLN levels were approximately 10 -fold lower, the resulting PLN/SERCA2 ratio in mouse atria was reduced by approximately 50 -fold compared with the ratio in ventricles (Figure 6B). Consequently, ISO/RO treatment induced only remote phosphorylation changes of PLN-S16. Overall, these data indicate a minor regulatory role of PLN for SERCA2 function in AMs (Figure 6B).

Since SERCA2 activity determines the SR transmembrane $\mathrm{Ca}^{2+}$ gradient underlying SR $\mathrm{Ca}^{2+}$ release, we measured steadystate $\mathrm{Ca}^{2+}$ transients during pacing. Consistent with previous studies (16), $\mathrm{Ca}^{2+}$ transients in mouse AMs showed significantly higher amplitudes and faster rise and decay rates compared with VMs (Supplemental Figure 13). We assessed the SR Ca ${ }^{2+}$ load by caffeine application (10 $\mathrm{mM}$ ) and found an approximately 2-fold higher amplitude of the $\mathrm{Ca}^{2+}$ transient in AMs versus that in VMs (Figure 6D), consistent with previous observations in rat AMs (16). 
A
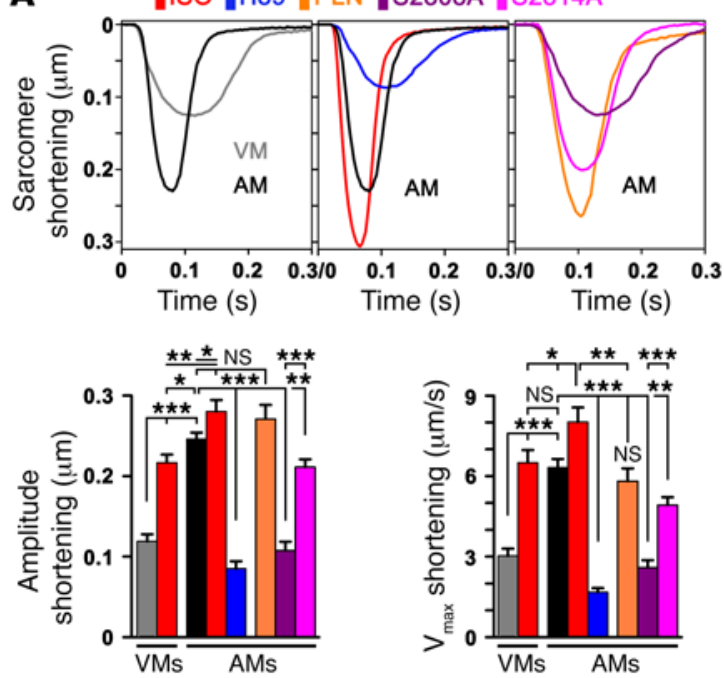

C

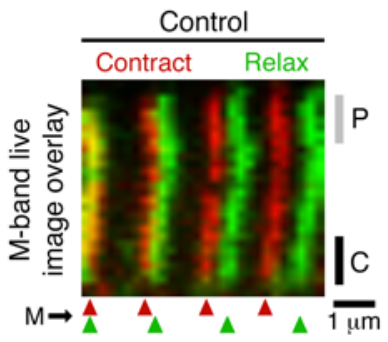

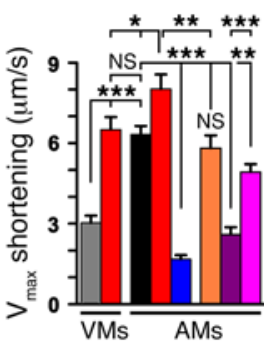

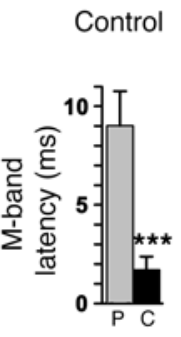

B

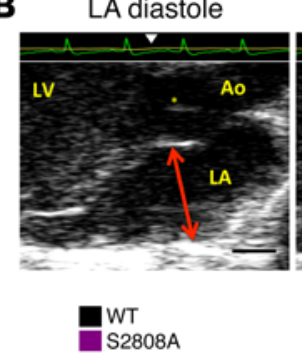

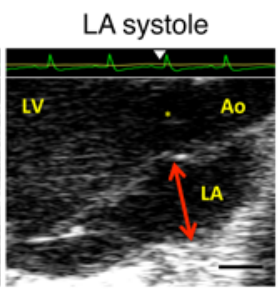

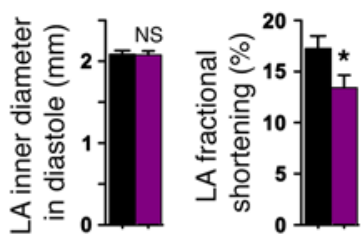

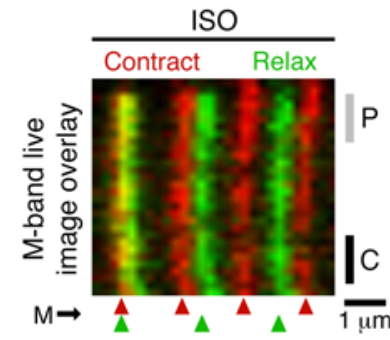

ISO

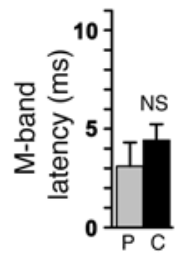

Figure 7. Catecholaminergic RyR2 cluster recruitment regulates sarcomere shortening and atrial contractility. (A) Representative AM sarcomere length traces during unloaded shortening; colors at top indicate the different conditions; VMs were used as a reference. The same WT AM control trace (black) is presented twice for comparison. WT AMs were treated with ISO $(1 \mu \mathrm{M})$ or H89 $(1 \mu \mathrm{M})$ and compared with RyR2-S2808A ${ }^{+/+}$-knockin, RyR2-S2814A ${ }^{+/+}-\mathrm{knock}^{-}$ in, or PLN-KO $\left(P / n^{-1-}\right)$ strains. Bar graphs summarizing maximal sarcomere shortening amplitude and velocity $\left(\mathrm{V}_{\max }\right)$ for the indicated conditions. Data are representative of 29 VMs and 59 AM WT controls; ISO, 13 AMs; H89, 33 AMs; S2808A, 44 AMs; S2814A, 36 AMs; and PLN, 17 AMs. ${ }^{*} P<0.05$, ${ }^{* *} P<0.01$, and ${ }^{* *} P<0.001$, by Student's $t$ test. (B) LA echocardiogram showing normal LA diameters in diastole versus systole (arrows) but significantly decreased fractional shortening in RyR2-S2808 $\mathrm{A}^{+/}$mice. An electrocardiogram (ECG) recording (green) was used to time maximal atrial relaxation and contraction (triangles). $n=18$ WT mice and 19 RyR2-S2808A mice ${ }^{+/+}$. ${ }^{*} P<0.05$, by Student's $t$ test. Ao, aorta; asterisk indicates the aortic valve. Scale bars: 1 mm. (C) Confocal live-image overlays were aligned to the left-most striation as indicated. The flexible nature of the sarcomeric M-bands was visualized during AM contraction (red) versus relaxation (green) using knockin mice expressing C-terminal-tagged titin-EGFP and peripheral versus central readouts as indicated. Bar graphs summarize the relative $\mathrm{M}$-band latency between peripheral $(P)$ versus central $(C) M$-band regions in control-versus ISO-treated (1 $\mu \mathrm{M})$ cells. $n=29$ control and 24 ISO AMs. ${ }^{* *} P<0.001$, by Student's $t$ test.

Both protein expression and $\mathrm{Ca}^{2+}$ transient data support a model of constitutively high SERCA2 function in atria. Hence, lowphos RyR2 cluster recruitment during catecholaminergic stimulation may represent a major and potentially cell-specific regulatory mechanism of $\mathrm{SR} \mathrm{Ca}^{2+}$ cycling in atria.

Catecholaminergic RyR2 cluster recruitment regulates atrial sarcomere shortening. The characteristic $\mathrm{Ca}^{2+}$ transient profiles we observed in AMs may support cell type-specific sarcomere shortening behavior. More specifically, rapid activation of ECC at baseline might be augmented by $\beta$-AR stimulation axially and transversally through faster recruitment of sarcomeres near lowphos RyR2 clusters. First, we measured the maximal sarcomere-shortening amplitude and velocity $\left(\mathrm{V}_{\max }\right)$ under baseline and ISO-stimulated conditions in mechanically unloaded WT cells. Sarcomere-shortening amplitude was significantly higher in ISO-treated AMs compared with that of control AMs or ISO-treated VMs (Figure 7A). Whereas ISO $(1 \mu \mathrm{M})$ significantly increased the shortening amplitude and $\mathrm{V}_{\max }$ in both VMs and AMs, the absolute level was always greater in AMs (Figure 7A). In contrast, PKA inhibition by H89 $(1 \mu \mathrm{M})$ decreased the sarcomere-shortening amplitude and $\mathrm{V}_{\max }$ compared with that observed in untreated AMs (Figure 7A).

To investigate PKA-dependent SR $\mathrm{Ca}^{2+}$ uptake, we studied AMs from PLN-KO ( $\left.\mathrm{Pln}^{-/}\right)$mice with constitutively upregulated SERCA2 function. While the shortening amplitude was not significantly different in $\mathrm{Pln}^{-/}$AMs compared with that of ISO-stimulated AMs, the $\mathrm{V}_{\max }$ was significantly smaller and similar to that of control WT AMs (Figure 7A). In summary, PLN ablation had no significant effect on sarcomere shortening compared with unstimulated AMs, consistent with the overall low atrial PLN protein expression levels in AMs.

To probe the functional role of RyR2 channel phosphorylation, we analyzed AMs from PKA phosphorylation-incompetent RyR2-S2808A ${ }^{+/+}$and CaMK phosphorylation-incompetent RyR2-S2814A $\mathrm{A}^{+/+}-$knockin mice. Compared with WT cells, both RyR2-S2808A ${ }^{+/+}$and RyR2-S2814A ${ }^{+/+}$cells exhibited significantly decreased shortening amplitude and $\mathrm{V}_{\max }$ under basal (Figure 7A) 
A

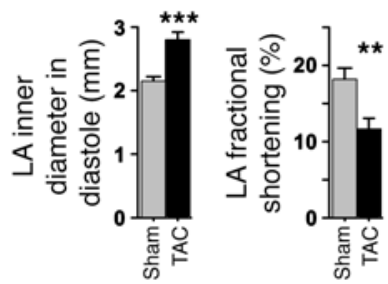

C

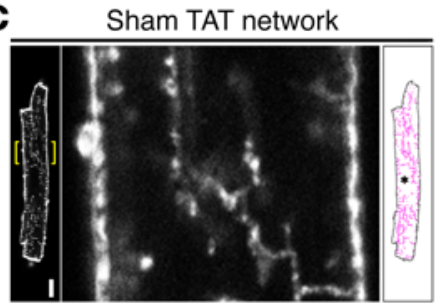

B

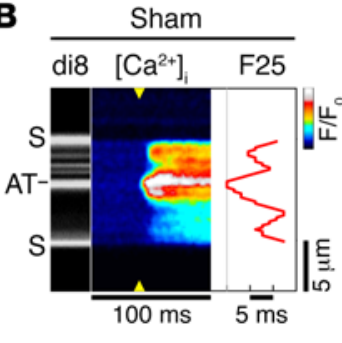

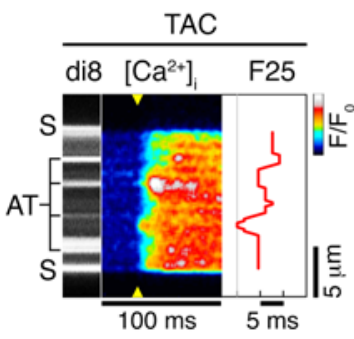

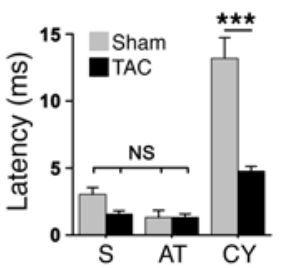

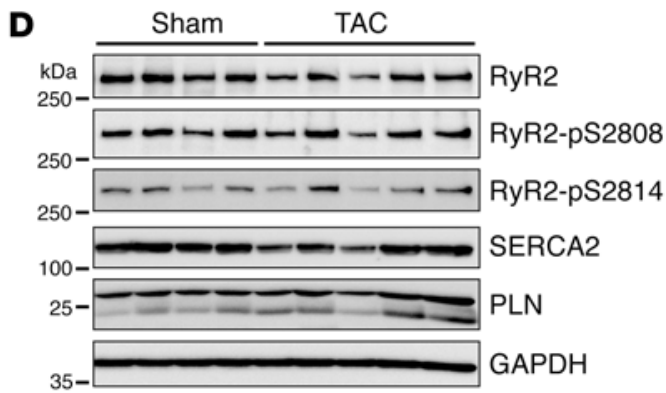
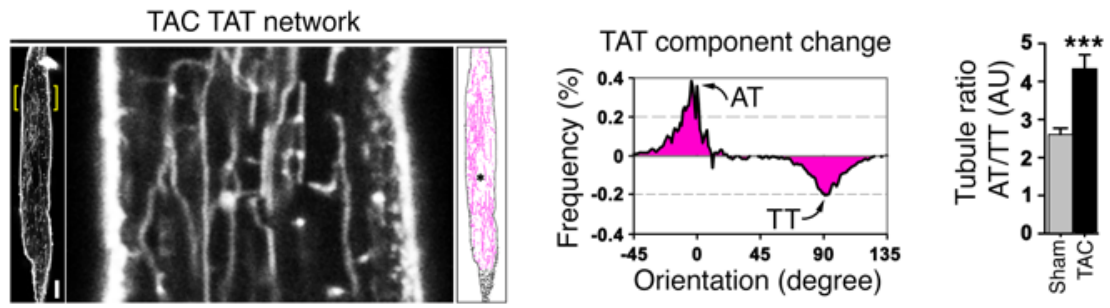

E

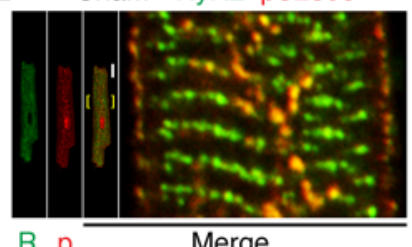

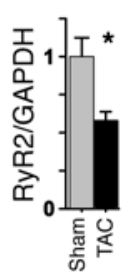

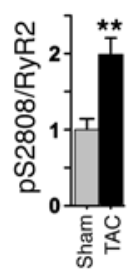

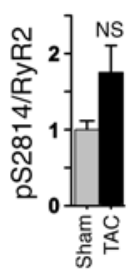
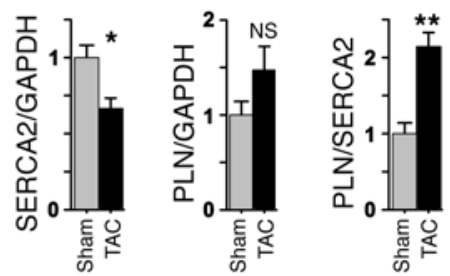

R p

Merge

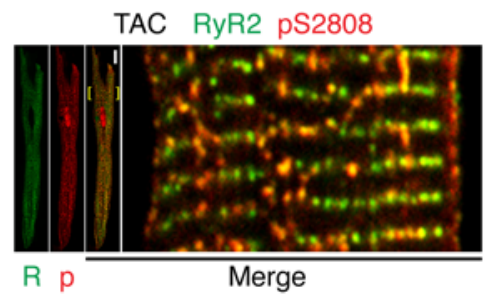

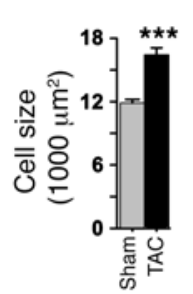

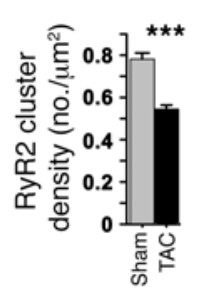

Figure 8. Atrial hypertrophy causes AT proliferation and faster $\mathrm{Ca}^{2+}$ release activation. (A) Echocardiography confirmed significant changes in atrial size and contractile function after TAC. $n=19$ sham mice and 21 TAC-treated mice. ${ }^{* *} P<0.01$ and ${ }^{* * *} P<0.001$, by Student's $t$ test. (B) Confocal line-scan images correlating local $\mathrm{Ca}^{2+}$ transient onset with membrane structures versus cytosolic locations in paced AM from the left atrium ( $\left.0.5 \mathrm{~Hz}\right)$. Compared with sham-treated mice, the post-TAC latency of SR Ca ${ }^{2+}$ release was significantly decreased at off-membrane CY locations. $n=16$ sham-treated AMs, 22 TAC AMs. ${ }^{* *} P<0.001$, by Student's $t$ test. (C) Confocal TAT imaging in live AM from the left atrium of sham or TAC hearts. After TAC, TAT skeletons (magenta) show proliferative changes, as quantified by TAT component analysis in hypertrophied LA AMs. Histogram subtraction (TAC - sham) demonstrated differential post-TAC changes: increased AT $\left(0^{\circ}\right)$ versus decreased TT components $\left(90^{\circ}\right)$. Bar graph summarizes significantly increased post-TAC AT/TT ratio (binning $\left.\pm 10^{\circ}\right) . n=34$ sham-treated AMs and 44 TAC-treated AMs from 3 to 4 hearts each. ${ }^{* * *} P<0.001$, by Student's $t$ test. Scale bars: $10 \mu \mathrm{m}$, magnification $\times 4$. (D) Immunoblots from atrial tissue lysates of sham- and TAC-treated hearts. Protein expression and phosphorylation changes are summarized by bar graphs. Blots are representative of 3 independent experiments. ${ }^{*} P<0.05$ and ${ }^{* *} P<0.01$, by Student's $t$ test. (E) Confocal images of AM from the left atrium showing increased highphos RyR2 cluster abundance in more numerous central-axial string-of-pearls in hypertrophied post-TAC as compared to sham-treated AM. Bar graphs summarize significance for an increase in cell size, a decrease in RyR2 cluster density, and an increase in the highphos RyR2-pS2808 cluster fraction after TAC. $n=33$ sham-treated and 46 TAC-treated AMs. ${ }^{* *} P<0.001$, by Student's $t$ test. Scale bars: $10 \mu \mathrm{m}$, magnification $\times 4$.

and ISO-stimulated $(1 \mu \mathrm{M})$ conditions (data not shown). While both RyR2-knockin models showed decreased shortening and $\mathrm{V}_{\max }$ under basal conditions, the absolute decrease was significantly greater in RyR2-S2808A ${ }^{+/+}$cells (Figure 7A). Given the more pronounced effect on sarcomere function in RyR2-S2808 $\mathrm{A}^{+/+}$cells, we used LA echocardiography to explore the functional relevance of highphos RyR2 cluster ablation in vivo in RyR2-S2808 $\mathrm{A}^{+/+}$mice. While age-matched RyR2-S2808A ${ }^{+/+}$mice with PKA phosphory- lation-incompetent RyR2 channels exhibited normal LA diastolic diameters, fractional shortening was decreased (Figure 7B). Hence, genetic ablation of RyR2 PKA phosphorylation is sufficient to cause significant loss of atrial function in vivo, presumably through depressed sarcomere shortening in AMs.

Since the M-band is generally considered a relatively elastic transversal sarcomere structure, we hypothesized that systolic M-band deformation could correlate with the $\mathrm{Ca}^{2+}$ transient pro- 
files observed in AMs. To assess this, we imaged EGFP-labeled M-band striations using AM from knockin mice that express a C-terminally tagged titin-EGFP fusion protein (38). We found a significant transversal latency difference between peripheral and central M-band deformations during sarcomere shortening in live AMs under baseline conditions (Figure 7C; compare Supplemental Videos 1 and 2 or Supplemental Videos 3 and 4). Consistent with transversal lowphos-to-highphos RyR2 cluster conversion, acute ISO $(1 \mu \mathrm{M})$ stimulation curtailed the M-band-specific latency difference (Figure 7C). The blunted M-band latency difference corresponded to the accelerated $\mathrm{Ca}^{2+}$ release spread observed in ISO/RO-stimulated AMs (Figure 6A).

We thus identified the following features of atrial sarcomere regulation: 1) axial sarcomere shortening is constitutively increased through tonic highphos RyR2 cluster regulation in AMs, contributing to rapid $\mathrm{Ca}^{2+}$ transient onset through AT-coupled highphos RyR2 clusters at baseline; 2) $\beta$-AR stimulation significantly increases sarcomere shortening and reduces transversal latency differences during sarcomere contraction.

Atrial hypertrophy causes AT proliferation, leading to accelerated cytosolic $\mathrm{Ca}^{2+}$ release. Atrial hypertrophy has been linked to depressed atrial contractility in patients and represents an important risk factor for AF, thromboembolic stroke, and HF. However, the mechanisms leading from atrial hypertrophy (frequently associated with systemically increased blood pressure) to contractile dysfunction, in particular prior to and/or independently of $\mathrm{AF}$, remain unclear. We used a model of transaortic constriction (TAC) in mice to induce hypertrophy of the LA. Four weeks after TAC, echocardiography confirmed significantly increased LA inner diameters and decreased fractional LA shortening (Figure 8A and Supplemental Table 2). Furthermore, increased hypertrophic wall thickening (left ventricular anterior wall thickness in diastole [LV AWTh]) and increased heart weight (HW/BW) were observed (Supplemental Table 2). Histological analysis confirmed that TAC induced significant LA hypertrophy and dilation, but showed no right atrial (RA) pathology (Supplemental Figure 14, A-C).

To explore potential atrial mechanisms of contractile dysfunction, we analyzed AT-dependent $\mathrm{Ca}^{2+}$ transient behavior using transversal line scanning of AM from the left atrium of sham-operated and TAC hearts. Hypertrophied AMs from TAC hearts showed more numerous AT structures and considerably compressed $\mathrm{Ca}^{2+}$ transient latency profiles, indicating accelerated cytosolic $\mathrm{Ca}^{2+}$ release in post-TAC AMs (Figure $8 \mathrm{~B}$ ). Analysis of the $\mathrm{Ca}^{2+}$ transient latency profiles $(\mathrm{dF} / \mathrm{dt})$ showed that off-membrane CY sites contributed significantly to the accelerated cytosolic $\mathrm{Ca}^{2+}$ release spread after TAC compared with that seen in sham AMs (Figure 8B). Hence, post-TAC cells are characterized by significantly accelerated cytosolic $\mathrm{Ca}^{2+}$ release through nonjunctional RyR2 cluster sites that are apparently activated through more numerous AT structures in substantially thicker AMs.

To elucidate hypertrophic changes in AT structures after TAC, we performed live-cell confocal membrane imaging. Hypertrophied AM from the left atrium showed an extensive proliferative adaptation of their TAT network compared with sham AMs (Figure $8 \mathrm{C})$. For comparative TAT network analysis, the difference integral of all TAT component orientations was plotted for post-TAC minus sham, highlighting an increase in AT $\left(0^{\circ}\right)$ and a decrease in TT $\left(90^{\circ}\right)$ components in hypertrophied AMs after TAC (Figure $8 \mathrm{C}$ ). Consequently, the post-TAC AT/TT ratio was significantly increased (Figure 8C). Notably, while atrial hypertrophy leads to select proliferation of AT components, this occurs on the background of significantly increased cell dimensions (Supplemental Figure 14D). Since AT structures couple highphos RyR2 clusters to rapid $\mathrm{Ca}^{2+}$ release in AMs, we next sought to analyze proteomic and in situ RyR2 cluster changes.

Immunoblotting showed significantly decreased post-TAC RyR2 protein levels (Figure 8D). Since RyR2 expression was not changed in LV tissue (data not shown), the observed post-TAC RyR2 channel downregulation appeared to be atria specific. Furthermore, PKA phosphorylation was significantly increased at the RyR2-S2808 phosphorylation site, but not at the CaMK phosphorylation site RyR2-S2814, in atrial tissue (Figure 8D). In contrast, 4 weeks after TAC in ventricular tissue, RyR2-S2814, but not RyR2-S2808, phosphorylation was substantially increased (data not shown), confirming an earlier study (39) and supporting the presence of atria-specific RyR2-S2808 phosphorylation changes. PKA hyperphosphorylation of RyR2 is known to increase the sensitivity for $\mathrm{Ca}^{2+}$ activation, which is generally consistent with altered cytosolic $\mathrm{SR} \mathrm{Ca}^{2+}$ release in post-TAC AMs (Figure 8B). Additionally, decreased SERCA2 expression increased the PLN/SERCA2 ratio and may have depressed post-TAC SR Ca ${ }^{2+}$ uptake in AMs (Figure 8D). Of note, increased SR Ca ${ }^{2+}$ release in the context of increased RyR2 phosphorylation and decreased $\mathrm{Ca}^{2+}$ uptake may increase the propensity for cytosolic $\mathrm{Ca}^{2+}$ overload at higher heart rates.

In atrial hypertrophy with increased post-TAC AT proliferation, a parallel increase of highphos RyR2 clusters occurred, along with more numerous axial-central strings heterogeneously distributed across thicker AM cells from the left atrium (Figure 8E). Using isolated AMs from the LA of TAC hearts, we confirmed a significantly increased post-TAC hypertrophic cell size compared with sham-treated cells (Figure 8E and Supplemental Figure 14D). However, despite the observed increase in highphos RyR2 cluster strings, the cell-wide density of all RyR2 clusters (both lowphos and highphos) was significantly decreased after TAC (Figure 8E), in agreement with the observed decrease in RyR2 protein expression levels. Yet, the AM-specific fraction of highphos RyR2-pS2808 clusters was significantly increased (Figure 8E). This indicates a relatively strong increase in highphos, at the cost of a reduced lowphos RyR2 cluster population and can explain the observed $\mathrm{Ca}^{2+}$ transient changes through earlier nonjunctional cluster recruitment, contributing to overall rapid $\mathrm{Ca}^{2+}$ transient onset, as observed throughout hypertrophied AMs (Figure 8B). Taken together, the activation of $\mathrm{SR} \mathrm{Ca}^{2+}$ release through more numerous AT structures and through an increased highphos RyR2 cluster fraction contributes to accelerated recruitment and earlier activation of adjacent nonjunctional lowphos clusters, indicating a sustained mechanism of subcellular $\mathrm{Ca}^{2+}$ transient adaptation in hypertrophied AMs, despite significantly decreased RyR2 channel expression.

\section{Discussion}

Here, we identify an atrial ECC "super-hub" mechanism that is based on a highly specialized membrane-protein network characterized by: 1) tubular membrane structures (ATs) with axial orientations that are 2) abundant in AMs and 3) connected to the 
surface membrane through sparse TT components; 4) significantly larger-diameter AT structures compared with TT structures in AMs; 5) AT-coupled large junctional RyR2 clusters that are highly phosphorylated by PKA and CaMK and arranged in axial stringof-pearls; 6) triggering by ATs of rapid $\mathrm{Ca}^{2+}$ release from axial highphos RyR2 cluster couplons in AMs via Cav1.2 channels; and both 7) rapid central $\mathrm{Ca}^{2+}$ transient onset and 8) central $\mathrm{Ca}^{2+}$ macro-sparks that are activated via junctional highphos RyR2 clusters at AT sites. While these observations present a robust intracellular context for rapid $\mathrm{Ca}^{2+}$ signaling in AMs, they also lay the foundation for novel and, most important, atria-specific regulatory ECC mechanisms. Given the relevance and functionality of the membrane-protein network detailed above, we propose a signaling super-hub model that is structurally based on unusually large AT and axial couplon dimensions and that may change our perspective on cell-specific control mechanisms of atrial $\mathrm{Ca}^{2+}$ signaling and contractile regulation.

Our observations are supported by previously described dedicated AM isolation and high-resolution imaging workflows (18, 40), which led to our finding of preserved centrally located AT structures within TAT networks. Since AMs are generally thinner than VMs, it has been proposed that TTs are not needed for ECC in these cells $(8,19)$. However, when we normalized the total TAT network length to the cell area, we found AMs with intermediate (Figure 1B) rather than low or zero TAT densities, as previously reported in rodents (8). Furthermore, we found voluminous AT structures that were electrically connected to the surface sarcolemmal membrane, presumably via more sparsely present TTs. While overall similar, TAT structures were shown previously in a minority of cells isolated from atria $(7,41)$. Here, we identify ATs as regularly present and specific structural components that are key to ECC in AMs.

Novel cell-specific properties: highly phosphorylated RyR2 clusters and SR load tune $\mathrm{Ca}^{2+}$ release at AT locations. In living AMs, STED revealed that central RyR2 clusters may be spaced more tightly along Z-lines than are those in VMs. We show that the vast majority of transversely oriented RyR2 cluster striations have no nearby TT membrane and are therefore not triggered by the opening of local LCC. In theory, an increased density of transverse RyR2 clusters would allow a CICR signal from the lateral cell surface to activate its nearest neighbor cluster more efficiently during the inward propagation of a $\mathrm{Ca}^{2+}$ wave, as shown by previous mathematical modeling (19). However, we found that cellular depolarization elicited $\mathrm{Ca}^{2+}$ transients significantly earlier at AT locations than at nonjunctional cytosolic cluster sites, or even at the AM surface. AM activation near the center of the cell has been observed and reported as irregular $\mathrm{Ca}^{2+}$ transients (7), but the physiological significance of this has not been appreciated, so that central RyR2 cluster ECC mechanisms remained undefined (42). Accordingly, the approximately $2-\mu \mathrm{m}$-wide gap separating surface-coupled peripheral RyR2 clusters from central RyR2 clusters was assumed to delay activation of the latter and to contribute to U-shaped $\mathrm{Ca}^{2+}$ activation in AMs (19). While we can confirm the peripheral RyR2 cluster gap in AMs (Figures 3, A and E), an exclusive dependence of ECC on peripheral clusters seems unlikely. Instead, deep inside $\mathrm{AM}, \mathrm{Ca}^{2+}$ release is activated via AT depolarization, which occurs approximately 2 times faster compared with clusters near the sur- face membrane. Since AT structures and axial junctions are ideally positioned to activate myofilaments at the AM center, highphos RyR2 cluster activation can efficiently support fast activation of central sarcomeres in AMs, which would be unlikely if U-shaped $\mathrm{Ca}^{2+}$ transients with a delay of approximately $100 \mathrm{~ms}$ (7) were to control ECC in AMs. Indeed, we found that central myofilaments are activated significantly earlier than are peripheral myofilaments, given the M-band striation changes in living AMs visualized by titin-EGFP knockin. Taken together, both the earlier activation of central $\mathrm{Ca}^{2+}$ release through ATs and the faster central sarcomere activation observed in AMs agree with the generally faster ECC of atrial muscle strips observed previously (12).

Our use of voltage-clamp and EGTA $(1 \mathrm{mM}) \mathrm{Ca}^{2+}$ buffering allows us to conclude that electrical transmission via the TAT network underlies the more rapid ECC activation of AT-coupled junctional RyR2 clusters. Here, we identify highly and stably phosphorylated RyR2 clusters, which are to a large degree associated with AT membranes. At the RyR2 clusters, combined PKA and CaMK phosphorylation of RyR2-S2808 and RyR2-S2814, respectively, significantly increases the sensitivity of the $\mathrm{Ca}^{2+}$-dependent activation of RyR2 (29, 30, 43). Consequently, junctional AT couplons are ideally positioned to rapidly initiate local $\mathrm{SR} \mathrm{Ca}^{2+}$ release, as evidenced by $\mathrm{Ca}^{2+}$ macro-sparks. Furthermore, we show that nonjunctional SR locations contribute a functionally important and economic form of "inotropic reserve," accessible through cAMP-dependent recruitment of lowphos RyR2 clusters and representing the first example, to our knowledge, of a differential regulatory mechanism of this protein in distinct SR domains.

We observed a high level of SR $\mathrm{Ca}^{2+}$ load in mouse AMs under baseline conditions, consistent with earlier observations (16). Within the context of highphos versus lowphos RyR2 clusters, a high SR $\mathrm{Ca}^{2+}$ content against the background of PLN-deficient AMs can explain the significantly larger central $\mathrm{Ca}^{2+}$ transient amplitude observed at baseline. Accordingly, we confirmed that SERCA2 protein levels were approximately 2 times higher in mouse atria than in ventricles, which was previously shown to stabilize $\mathrm{Ca}^{2+}$ cycling in ratAM (16). Importantly, minimal PLN protein levels in mouse atria resulted in a very low PLN/SERCA2 ratio. This is consistent with an earlier study showing that the transcription factor COUP-TFII suppresses PLN transcription in atria (44). Therefore, PKA-dependent recruitment of lowphos RyR2 clusters may represent a main inotropic $\mathrm{SR}$ regulatory mechanism in AMs. Indeed, PLN ablation did not significantly change the amplitude of sarcomere shortening in AMs from $\mathrm{Pln}^{-/-} \mathrm{KO}$ mice.

Using a simplified mathematical model of atrial $\mathrm{Ca}^{2+}$ sparks, we were able to reproduce the macro-spark behavior observed at the AM center, on the basis of stochastic RyR2 gating of lowphos and highphos clusters. As has been established before, the control of RyR2 sensitivity to cytosolic $\left[\mathrm{Ca}^{2+}\right]_{\mathrm{i}}$ appears to depend on multiple factors and interactions. Recent investigations suggest that $\mathrm{Ca}^{2+}$ in the SR lumen $\left(\left[\mathrm{Ca}^{2+}\right]_{j \mathrm{SR}}\right)$ may exert a nonlinear action on the sensitivity of the RyR2 clusters to be triggered $(45,46)$. Thus, a higher $\left[\mathrm{Ca}^{2+}\right]_{j \mathrm{SR}}$ can underlie $\mathrm{Ca}^{2+}$ sparks, with a greater total amount of $\mathrm{Ca}^{2+}$ released per average event. $\mathrm{Ca}^{2+}$ macro-sparks may facilitate the local propagation of CICR, originating from axial couplons. The atrial super-hub provides a new concept, on the background of predominant expression of fast $\alpha$-myosin heavy 
chain in atria, for both the rapid onset and extent of AM contraction, the potential for physiological regulation, and altered highphos RyR2 cluster function in hypertrophy.

Alterations of axial couplon function in atrial disease. In rabbit $\mathrm{AM}$ after 5 days of rapid atrial pacing RyR2 protein expression was reduced by $50 \%$ (4), similar to our observations in mouseAM postTAC (Figure 8D). This has given rise to the concept of a physiological antiarrhythmic adaptation ( $\mathrm{Ca}^{2+}$-signaling silencing), attributed to a failure of central CICR due to reduced RyR2 channel numbers per cluster and despite RyR2-S2808 hyperphosphorylation (4). In contrast, after TAC, we found an additional decrease of approximately $30 \%$ in RyR 2 cluster density, yet identified an increase of highphos RyR2 clusters in more numerous strings, which contributed to an approximately 2-fold increase in the highphos RyR2 cluster fraction. This suggests the presence of an adaptive mechanism that sustains cytosolic $\mathrm{Ca}^{2+}$ release through AT-coupled central RyR2 clusters after TAC, in stark contrast to the reported tachypacing-induced failure of central $\mathrm{Ca}^{2+}$ signal propagation (4). As hypertrophy and reduced contractility were identified in canine atria as early as 2 days after pacing (5), it will be important for future studies to explore the precise time course of transition from normal to hypertrophied atria. In contrast to our post-TAC model, a tachypacing HF model of persistent AF in sheep showed a complete loss of atrial TAT structures, consistent with advanced HF $(20,47)$. While we examined the LA response to TAC-induced atrial hypertrophy in the absence of any obvious arrhythmias (data not shown), the adaptive response was a profound increase in AT components, highphos RyR2 clusters, and increased nonjunctional $\mathrm{Ca}^{2+}$ release.

Clinical relevance of atrial super-hubs. The use of STED nanoscopy and ET, combined with membrane-preserving workflows, has enabled visualization of AT structures throughout AMs. Instead of the TAT being absent in AMs as previously suggested $(6,20)$, we found that a robust and functionally relevant tubular membrane system was present in every AM studied. In mouse and human AMs, AT membrane structures are an essential component of axial couplons that contain extended RyR2 clusters. The functions of this complex assembly, described as super-hubs, are likely to play important roles in modulating atrial ECC, distinct from classic couplons in VMs. In contrast, RyR2 PKA phosphorylation is significantly increased in patients with chronic AF (33) and after short-term tachypacing (4). Both RyR2 single-channel function and PKA phosphorylation were partially normalized in ventricular samples from HF patients treated with $\beta$-AR blockers (48). Since $\beta$-AR blockers are used in approximately $90 \%$ of patients with AF (2), our finding of a super-hub mechanism of contractile regulation in AMs through highphos RyR2 clusters may be instrumental in specifically targeting the (side) effects of GPCR-targeted drug compounds including $\beta$-AR blockers or currently available CaMK inhibitors on atrial contractility. Future work will need to examine the diverse functional roles of axial couplons and changes in RyR2 cluster regulation of hypertrophy in atrial cells, tissues, and the heart in vivo. The causal relation between highphos RyR2 clusters and rapid atrial contractile activation, identified through RyR2 phosphorylation-incompetentknockin animals, and the sustained structural and metabolic adaptation mechanisms in hypertrophy strengthen the rationale for the identification of early atrial disease mechanisms and, ultimately, for AM-directed treatment approaches.

\section{Methods}

Additional information is provided in the Supplemental Methods.

Mouse cells. AMs and VMs were isolated from adult mouse hearts using collagenase and protocols tuned to preserve subcellular structural integrity, as previously described $(9,18)$. RyR2-S2814A $\mathrm{A}^{+/+}$-knockin mice were provided by Xander H.T. Wehrens (Baylor College of Medicine, Houston, Texas, USA); and PLN-KO mice were provided by Despina Sanoudou (University of Athens, Athens, Greece). Unless indicated otherwise, the genetic background was C57BL/6N.

Human cells and tissues. Atrial tissue samples were obtained from patients with sinus rhythm during bypass surgery, following written informed consent from the patients. Cell isolation $(n=5)$ occurred from atrial tissue by protocols described previously (49). Cells were plated on laminin-coated coverslips for immunofluorescence staining. For IHC, LA tissue was fixed in $4 \%$ paraformaldehyde (PFA), embedded in paraffin, cut into $4-\mu \mathrm{m}$-thick sections, deparaffinized, rehydrated, and antigens unmasked in $10 \mathrm{mM}$ sodium-citrate buffer prior to Ab incubation.

Live-cell confocal microscopy. Confocal images were acquired with a Zeiss LSM 710 NLO microscope using a Plan-Apochromat 63x/1.40 oil objective and a pixel size of $100 \mathrm{~nm}$. Myocytes attached to laminin-coated slides were stained with $50 \mu \mathrm{M}$ di-8-ANEPPS (Molecular Probes) for 15 minutes, washed twice, excited at $488 \mathrm{~nm}$, and emitted light detected above $510 \mathrm{~nm}$ as previously described (9). TAT networks in AMs and VMs were visualized using identical settings. Intracellular $\mathrm{Ca}^{2+}$ imaging with fluo-4-AM was combined with di-8-ANEPPS as described previously (9). For $\mathrm{Ca}^{2+}$ spark imaging and signal analysis, we used previously established protocols (50). Further details on the TAT component analysis, $\mathrm{Ca}^{2+}$ transient analysis, or patch-clamping are available in the Supplemental Methods.

Live cell STED microscopy. For super-resolution imaging of select TAT membrane tubules, we used a customized STED system and Matlab (The MathWorks) algorithms as described previously $(9,18)$. Further details are provided in the Supplemental Methods.

ET. AT and TT structures and isolated AM were preserved by high-pressure freezing with an HPM100 system (Leica) as described previously (51). ET at the Electron Microscopy core facility at the European Molecular Biology Laboratory (EMBL) Heidelberg (Heidelberg, Germany) and at the EM services of the University of Colorado Boulder (Boulder, Colorado, USA) was performed as described previously (23).

Pharmacological assays. AMs plated on laminin-coated coverslips were pretreated with $10 \mu \mathrm{M}$ RO dissolved in DMSO (Sigma-Aldrich) for 3 minutes, followed by $1 \mu \mathrm{M}$ ISO (Sigma-Aldrich) for 10 minutes (ISO/RO). For Western blot analysis, mouse hearts were Langendorff perfused as described above, followed by immediate tissue freezing. For acute $\beta$-AR stimulation, $1 \mu \mathrm{M}$ ISO was used if not stated otherwise. For in situ PKA inhibition, AMs were incubated for at least 15 minutes with H89 (Sigma-Aldrich). For in situ CaMK inhibition, cells were treated with $5 \mu \mathrm{M}$ AIP (Sigma-Aldrich) for 30 minutes.

FRET measurements. In AMs from mice expressing the Epac1camps FRET biosensor, intracellular cAMP was assessed during 0.1 $\mu \mathrm{M}$ ISO treatment as previously described (36). Additional details are provided in the Supplemental Methods.

Sarcomere shortening. Sarcomere shortening measurements used high optical contrast imaging according to the manufacturer's instruc- 
tions (IonOptix). To measure transversal sarcomere M-band changes in AMs, we developed a new confocal imaging strategy using previously described GFP-tagged titin-knockin mice (38). Additional details are provided in the Supplemental Methods.

Immunofluorescence microscopy. Cells on coverslips were fixed with 4\% PFA and permeabilized with $0.2 \%$ Triton. Cells were processed for immunofluorescence microscopy as described previously $(9,29)$. Confocal fluorescence images were obtained with a Zeiss LSM 710 NLO and super-resolution images with a Leica TCS STED laser-scanning microscope. Fluorophores were excited at $458 \mathrm{~nm}, 488 \mathrm{~nm}, 514$ $\mathrm{nm}, 561 \mathrm{~nm}, 580 \mathrm{~nm}$, or $633 \mathrm{~nm}$, and emission of dual or triple fluorophore combinations detected. All primary Abs and dilutions are summarized in Supplemental Table 3. RyR2-pS2814 Abs were provided by Xander H.T. Wehrens (Baylor College of Medicine, Houston, Texas, USA). Images were processed using ImageJ/Fiji (NIH) as described previously (9, 18). RyR2, RyR2-pS2808, RyR2-pS2814, Cav1.2, and Cav3 signals in AMs and NND relations were analyzed with macros using Fiji. Further details are provided in the Supplemental Methods.

PCR. Refer to the Supplemental Methods for a modified genotyping protocol involving the RyR2-S2808 $\mathrm{A}^{+/+}$-knockin mice described previously (29).

Western blots. Protein extracts from cardiac tissue lysates were prepared as described previously $(9,40)$. The primary Abs used are listed in Supplemental Table 3 and the complete gels in Supplemental Figure 10.

TAC and histology. TAC was used to induce LA hypertrophy in WT mice according to previously described protocols (52). Additional details are provided in the Supplemental Methods.

Echocardiography. Atrial dimensions were measured using a left parasternal long-axis view at the level of the aortic valve tip during simultaneous surface electrocardiogram recording (Vevo 2100; VisualSonics).

Statistics. Statistical analyses were performed with Microsoft Excel, GraphPad Prism (version 5.01), and SigmaPlot (Systat Software). Differences were analyzed by unpaired 2-tailed Student's $t$ test, Mann-Whitney $U$ test, or ANOVA. Data are presented as the mean \pm SEM unless otherwise indicated. $P$ values $<0.05$ were accepted to indicate statistical significance.

Study approval. This study was carried out according to guidelines for the care and use of laboratory animals following Direc- tive 2010/63/EU of the European Parliament and of the Council of the European Union, Strasbourg, France and in keeping with NIH guidelines. All animal procedures were reviewed by the IACUC and approved by the Veterinarian State Authority (LAVES, Oldenburg, Germany). All protocols were approved by the ethics committee of the University Medical Center Göttingen.

\section{Author contributions}

GSBW, PK, SB, SEL, TK, and WJL designed the studies. EARZ, EH, EW, GH, GSBW, KG, M Dura, M Didié, MG, PK, SB, SEL, VON, TK, and WJL performed the research and analyzed the data. PK, SB, SEL, and WJL wrote the manuscript, and all authors contributed to the final version. CWW performed research and analyzed the data.

\section{Acknowledgments}

We are grateful for the excellent technical assistance of Birgit Schumann, Brigitte Korff, and Kirsten Koschel; to Carolin Wichmann for help with high-pressure freezing sample preparation; and to the staff of the Electron Microscopy core facility at EMBL Heidelberg and the Boulder EM Services core facility at the University of Colorado. Human samples were provided by Samuel Sosalla (University Medical Center Göttingen, Göttingen, Germany). This work received support from Deutsche Forschungsgemeinschaft SFB1002 projects A09 (to SEL), B05 (to SEL), A01 (to VON), Z (to $\mathrm{GH}$ ), and service projects $\mathrm{SO} 1$ and $\mathrm{SO} 2$; the German Cardiac Society supporting the doctoral thesis of SB; in support of the doctoral thesis of SB; and the British Heart Foundation (fellowships for EARZ and PK). This research has also received funding from the European Community's Seventh Framework Program grant agreement HEALTH-F2-2009-241526, EUTrigTreat (to SEL and WJL) and the European Research Council (ERC) Advanced Grant CardioNect (to PK).

Address correspondence to: Stephan E. Lehnart, University Medical Center Goettingen, Clinic of Cardiology and Pulmonology, Robert-Koch-Str. 40, 37075 Goettingen, Germany. Phone: 49.551.39.10575; E-mail: slehnart@med.uni-goettingen.de.
1. Yiin GS, et al. Age-specific incidence, outcome, cost, and projected future burden of atrial fibrillation-related embolic vascular events: a population-based study. Circulation. 2014;130(15):1236-1244.

2. Nabauer M, et al. The Registry of the German Competence NETwork on Atrial Fibrillation: patient characteristics and initial management. Europace. 2009;11(4):423-434.

3. Akar JG, et al. Intracellular chloride accumulation and subcellular elemental distribution during atrial fibrillation. Circulation. 2003;107(13):1810-1815.

4. Greiser M, et al. Tachycardia-induced silencing of subcellular $\mathrm{Ca} 2+$ signaling in atrial myocytes. J Clin Invest. 2014;124(11):4759-4772.

5. Ramadeen A, et al. N-3 polyunsaturated fatty acid supplementation does not reduce vulnerability to atrial fibrillation in remodeling atria. Heart Rhythm. 2012;9(7):1115-1122.e4.
6. McNutt NS, Fawcett DW. The ultrastructure of the cat myocardium. II. Atrial muscle. J Cell Biol. 1969;42(1):46-67.

7. Kirk MM, et al. Role of the transverse-axial tubule system in generating calcium sparks and calcium transients in rat atrial myocytes. J Physiol (Lond). 2003;547(Pt 2):441-451.

8. Dibb KM, Clarke JD, Eisner DA, Richards MA, Trafford AW. A functional role for transverse (t-) tubules in the atria. JMol Cell Cardiol. 2013;58:84-91.

9. Wagner E, et al. Stimulated emission depletion live-cell super-resolution imaging shows proliferative remodeling of T-tubule membrane structures after myocardial infarction. Circ Res. 2012;111(4):402-414.

10. Song LS, Sobie EA, McCulle S, Lederer WJ, Balke $\mathrm{CW}$, Cheng $\mathrm{H}$. Orphaned ryanodine receptors in the failing heart. Proc Natl Acad Sci U S A. 2006;103(11):4305-4310.

11. Crossman DJ, et al. T-tubule disease: Relation- ship between t-tubule organization and regional contractile performance in human dilated cardiomyopathy. JMol Cell Cardiol. 2015;84:170-178.

12. Lüss I, et al. Expression of cardiac calcium regulatory proteins in atrium $\mathrm{v}$ ventricle in different species. JMol Cell Cardiol. 1999;31(6):1299-1314.

13. Brandenburg S, Arakel EC, Schwappach B, Lehnart SE. The molecular and functional identities of atrial cardiomyocytes in health and disease. Biochim Biophys Acta. 2016; 1863(7 Pt B):1882-1893.

14. Greiser M, Lederer WJ, Schotten U. Alterations of atrial $\mathrm{Ca}(2+)$ handling as cause and consequence of atrial fibrillation. Cardiovasc Res. 2011;89(4):722-733.

15. Carl SL, et al. Immunolocalization of sarcolemmal dihydropyridine receptor and sarcoplasmic reticular triadin and ryanodine receptor in rabbit ventricle and atrium. J Cell Biol. 1995;129(3):673-682. 
16. Walden AP, Dibb KM, Trafford AW. Differences in intracellular calcium homeostasis between atrial and ventricular myocytes. J Mol Cell Cardiol. 2009;46(4):463-473.

17. Hüser J, Lipsius SL, Blatter LA. Calcium gradients during excitation-contraction coupling in cat atrial myocytes. JPhysiol (Lond). 1996; 494 (Pt 3):641-651.

18. Wagner E, Brandenburg S, Kohl T, Lehnart SE. Analysis of tubular membrane networks in cardiac myocytes from atria ventricles. JVis Exp. 2014;(92):e51823.

19. Thul R, Coombes S, Roderick HL, Bootman MD. Subcellular calcium dynamics in a whole-cell model of an atrial myocyte. Proc Natl Acad Sci U S A. 2012;109(6):2150-2155.

20. Dibb KM, et al. Characterization of an extensive transverse tubular network in sheep atrial myocytes and its depletion in heart failure. Circ Heart Fail. 2009;2(5):482-489.

21. Frisk M, Koivumäki JT, Norseng PA, Maleckar MM, Sejersted OM, Louch WE. Variable t-tubule organization and $\mathrm{Ca} 2+$ homeostasis across the atria. Am J Physiol Heart Circ Physiol. 2014;307(4):H609-H62O.

22. Glukhov AV, et al. Direct evidence for microdomain-specific localization and remodeling of functional L-type calcium channels in rat and human atrial myocytes. Circulation. 2015;132(25):2372-2384.

23. Rog-Zielinska EA, Johnston CM, O'Toole ET, Morphew M, Hoenger A, Kohl P. Electron tomography of rabbit cardiomyocyte threedimensional ultrastructure. Prog Biophys Mol Biol. 2016;121(2):77-84.

24. Wong J, et al. Nanoscale distribution of ryanodine receptors and caveolin-3 in mouse ventricular myocytes: dilation of t-tubules near junctions. Biophys J. 2013;104(11):L22-L24.

25. Mackenzie L, Roderick HL, Berridge MJ, Conway SJ, Bootman MD. The spatial pattern of atrial cardiomyocyte calcium signalling modulates contraction. JCell Sci. 2004;117(Pt 26):6327-6337.

26. Nikolaev VO, et al. Beta2-adrenergic receptor redistribution in heart failure changes cAMP compartmentation. Science. 2010;327(5973):1653-1657.

27. Gao T, Puri TS, Gerhardstein BL, Chien AJ, Green RD, Hosey MM. Identification and subcellular localization of the subunits of L-type calcium channels and adenylyl cyclase in cardiac myocytes. J Biol Chem. 1997;272(31):19401-19407.

28. Lehnart SE, et al. Phosphodiesterase $4 \mathrm{D}$ deficiency in the ryanodine-receptor complex promotes heart failure and arrhythmias. Cell. 2005;123(1):25-35.

29. Wehrens XH, Lehnart SE, Reiken S, Vest JA, Wronska A, Marks AR. Ryanodine receptor/ calcium release channel PKA phosphorylation: a critical mediator of heart failure progression. Proc Natl Acad Sci U S A. 2006;103(3):511-518.

30. Wehrens XH, Lehnart SE, Reiken SR, Marks AR. $\mathrm{Ca} 2+/$ calmodulin-dependent protein kinase II phosphorylation regulates the cardiac ryanodine receptor. Circ Res. 2004;94(6):e61-e70.

31. Chelu MG, et al. Calmodulin kinase II-mediated sarcoplasmic reticulum $\mathrm{Ca} 2+$ leak promotes atrial fibrillation in mice. J Clin Invest. 2009;119(7):1940-1951.

32. Xie W, et al. Mitochondrial oxidative stress promotes atrial fibrillation. Sci Rep. 2015;5:11427.

33. Vest JA, et al. Defective cardiac ryanodine receptor regulation during atrial fibrillation. Circulation. 2005;111(16):2025-2032.

34. Voigt N, et al. Cellular and molecular mechanisms of atrial arrhythmogenesis in patients with paroxysmal atrial fibrillation. Circulation. 2014;129(2):145-156.

35. Wescott AP, Jafri MS, Lederer WJ, Williams GS. Ryanodine receptor sensitivity governs the stability and synchrony of local calcium release during cardiac excitation-contraction coupling. J Mol Cell Cardiol. 2016;92:82-92.

36. Calebiro D, et al. Persistent cAMP-signals triggered by internalized G-protein-coupled receptors. PLoS Biol. 2009;7(8):e1000172.

37. Agarwal SR, Clancy CE, Harvey RD. Mechanisms Restricting diffusion of intracellular cAMP. Sci Rep. 2016;6:19577.

38. da Silva Lopes K, Pietas A, Radke MH, Gotthardt $M$. Titin visualization in real time reveals an unexpected level of mobility within and between sarcomeres. J Cell Biol. 2011;193(4):785-798.

39. Respress JL, et al. Role of RyR2 phosphorylation at S2814 during heart failure progression. Circ Res. 2012;110(11):1474-1483.

40. Arakel EC, et al. Tuning the electrical properties of the heart by differential trafficking of KATP ion channel complexes. J Cell Sci. 2014; 127(Pt 9):2106-2119.

41. Smyrnias I, Mair W, Harzheim D, Walker SA, Roderick HL, Bootman MD. Comparison of the T-tubule system in adult rat ventricular and atrial myocytes, and its role in excitation-contraction coupling and inotropic stimulation. Cell Calcium. 2010;47(3):210-223.

42. Woo SH, Cleemann L, Morad M. Diversity of atrial local $\mathrm{Ca} 2+$ signalling: evidence from 2-D confocal imaging in Ca2+-buffered rat atrial myocytes. JPhysiol (Lond). 2005;567(Pt 3):905-921.

43. Shan J, et al. Phosphorylation of the ryanodine receptor mediates the cardiac fight or flight response in mice. J Clin Invest. 2010;120(12):4388-4398.

44. Wu SP, et al. Atrial identity is determined by a COUP-TFII regulatory network. Dev Cell. 2013;25(4):417-426.

45. Williams GS, Chikando AC, Tuan HT, Sobie EA, Lederer WJ, Jafri MS. Dynamics of calcium sparks and calcium leak in the heart. Biophys J. 2011;101(6):1287-1296.

46. Walker MA, et al. Superresolution modeling of calcium release in the heart. Biophys J. 2014;107(12):3018-3029.

47. Lenaerts I, et al. Ultrastructural and functional remodeling of the coupling between $\mathrm{Ca} 2+$ influx and sarcoplasmic reticulum $\mathrm{Ca} 2+$ release in right atrial myocytes from experimental persistent atrial fibrillation. Circ Res. 2009;105(9):876-885.

48. Reiken S, et al. Beta-blockers restore calcium release channel function and improve cardiac muscle performance in human heart failure. Circulation. 2003;107(19):2459-2466.

49. Voigt N, Zhou XB, Dobrev D. Isolation of human atrial myocytes for simultaneous measurements of $\mathrm{Ca} 2+$ transients membrane currents. JVis Exp. 2013;(77):e50235.

50. Lehnart SE, et al. Leaky Ca2+ release channel/ryanodine receptor 2 causes seizures and sudden cardiac death in mice. JClin Invest. 2008;118(6):2230-2245.

51. Vogl C, et al. Unconventional molecular regulation of synaptic vesicle replenishment in cochlear inner hair cells. J Cell Sci. 2015;128(4):638-644.

52. Toischer K, et al. Differential cardiac remodeling in preload versus afterload. Circulation. 2010;122(10):993-1003. 